\title{
Local anaesthetic infiltration for peri-operative pain control in total hip and knee replacement: systematic review and meta-analyses of short- and long-term effectiveness
}

Elsa MR Marques ${ }^{1 *}$, Hayley E Jones ${ }^{1}$, Karen T Elvers $^{2}$, Mark Pyke $^{3}$, Ashley W Blom² and Andrew D Beswick ${ }^{2}$

\begin{abstract}
Background: Surgical pain is managed with multi-modal anaesthesia in total hip replacement (THR) and total knee replacement (TKR). It is unclear whether including local anaesthetic infiltration before wound closure provides additional pain control.

Methods: We performed a systematic review of randomised controlled trials of local anaesthetic infiltration in patients receiving THR or TKR. We searched MEDLINE, Embase and Cochrane CENTRAL to December 2012. Two reviewers screened abstracts, extracted data, and contacted authors for unpublished outcomes and data. Outcomes collected were post-operative pain at rest and during activity after 24 and 48 hours, opioid requirement, mobilisation, hospital stay and complications. When feasible, we estimated pooled treatment effects using random effects meta-analyses.

Results: In 13 studies including 909 patients undergoing THR, patients receiving local anaesthetic infiltration experienced a greater reduction in pain at 24 hours at rest by standardised mean difference (SMD) -0.61 $(95 \% \mathrm{Cl}-1.05,-0.16 ; \mathrm{p}=0.008)$ and by SMD $-0.43(95 \% \mathrm{Cl}-0.78-0.09 ; \mathrm{p}=0.014)$ at 48 hours during activity. In TKR, diverse multi-modal regimens were reported. In 23 studies including 1439 patients undergoing TKR, local anaesthetic infiltration reduced pain on average by SMD $-0.40(95 \% \mathrm{Cl}-0.58,-0.22 ; \mathrm{p}<0.001)$ at 24 hours at rest and by SMD $-0.27(95 \% \mathrm{Cl}-0.50,-0.05 ; \mathrm{p}=0.018)$ at 48 hours during activity, compared with patients receiving no infiltration or placebo. There was evidence of a larger reduction in studies delivering additional local anaesthetic after wound closure. There was no evidence of pain control additional to that provided by femoral nerve block.

Patients receiving local anaesthetic infiltration spent on average an estimated $0.83(95 \% \mathrm{Cl} 1.54,0.12 ; \mathrm{p}=0.022)$ and $0.87(95 \% \mathrm{Cl} 1.62,0.11 ; \mathrm{p}=0.025)$ fewer days in hospital after THR and TKR respectively, had reduced opioid consumption, earlier mobilisation, and lower incidence of vomiting.

Few studies reported long-term outcomes.
\end{abstract}

Conclusions: Local anaesthetic infiltration is effective in reducing short-term pain and hospital stay in patients receiving THR and TKR. Studies should assess whether local anaesthetic infiltration can prevent long-term pain. Enhanced pain control with additional analgesia through a catheter should be weighed against a possible infection risk.

Keywords: Hip replacement, Knee replacement, Anaesthesia, Systematic review, Meta-analysis

\footnotetext{
* Correspondence: e.marques@bristol.ac.uk

'School of Social and Community Medicine, University of Bristol, Canynge Hall, 39 Whatley Road, Bristol BS8 2PS, UK

Full list of author information is available at the end of the article
} 


\section{Background}

Total hip replacement (THR) and total knee replacement (TKR) are widely used to treat diseased and damaged joints. In 2012 there were 75,366 primary THR and 76,497 primary TKR procedures recorded in the $\mathrm{Na}$ tional Joint Registry for England, Wales and Northern Ireland [1]. In the USA in 2010, the estimated numbers of THR and TKR procedures performed were 332,000 and 719,000, respectively [2].

Pain is the primary indication for THR and TKR and many preparatory, surgical and rehabilitation strategies target reduction in pain. However, both short- and longterm pain after THR and TKR are common [3-5]. Perioperative pain is managed with multi-modal analgesia with additive or synergistic effects [6]. Regimens aim to achieve good pain relief immediately after surgery while allowing for early mobilisation and hospital discharge. Other methods such as spinal and epidural anaesthetics and the use of opioids may preclude early mobilisation and rehabilitation $[7,8]$.

Pain management by infusion of local anaesthetic into wounds has been evaluated in diverse surgical procedures. In their systematic review, Liu and colleagues noted improved pain, reduced opioid use and side effects, increased patient satisfaction, and shorter hospital stay in patients receiving local anaesthetic infiltration [9]. Only one study included patients with THR or TKR [10], but further evaluations have been reported [11]. More recent meta-analyses in abdominal surgery [12], and lumbar spine surgery [13], have questioned the clinical value of local anaesthetic wound infiltration.

Using systematic review methods and meta-analysis, our objective was to synthesise evidence from randomised controlled trials (RCTs) evaluating the effectiveness of peri-operative local anaesthetic infiltration for pain control in patients with THR and TKR. Pain outcomes were considered along with post-operative opioid requirement, mobilisation, hospital stay and complications.

\section{Methods}

We identified RCTs using methods described in the Cochrane handbook of systematic reviews of interventions [14]. The review was conducted in accordance with PRISMA guidelines [15] and a checklist is included as Additional file 1. This review builds on a previous literature review, without a further formal protocol published [11].

\section{Search strategy and selection criteria}

We searched MEDLINE and Embase on OvidSP and Cochrane CENTRAL to $11^{\text {th }}$ December 2012. The search strategy covered RCTs, anaesthesia and analgesia, and THR and TKR terms (Table 1). We tracked citations of key articles in ISI Web of Science [10,16-19], and checked reference lists. Two reviewers scanned abstracts and titles, acquired potentially relevant articles, and decided on inclusion based on pre-specified criteria, with disputes resolved by other authors.

We included RCTs of patients with primary unilateral THR or TKR receiving local anaesthetic infiltration before wound closure compared with patients receiving no local anaesthetic infiltration or placebo. We also included studies comparing local anaesthetic infiltration with other forms of analgesia and studies with additional post-wound closure delivery of analgesics through catheters and injections. We excluded studies with interventions exclusively after wound closure and studies in patients receiving hip hemiarthroplasty or unicompartmental TKR. No language restrictions were applied and translations were made by colleagues as required.

\section{Data collection and extraction}

Articles and inclusion/exclusion decisions were catalogued in Endnote X5. Data were extracted on to piloted forms and an Excel spreadsheet in duplicate. Authors were contacted for unpublished outcomes and missing data.

Information was extracted on study characteristics; participant characteristics; anaesthesia procedures common to randomised groups; intervention (content of infiltrate, timing and volume); additional intervention group treatments; and control group treatment including placebo and alternative analgesia regimens.

\section{Outcomes}

Outcomes studied were pain at rest or during activity at 24 and 48 hours after surgery, opioid consumption, mobilisation, length of hospital stay in days, and long-term pain and function. Serious complications recorded were altered state of consciousness, cardiovascular complications requiring treatment, central nervous system toxicity, dysarthria, dyspnoea, major surgical complications, pneumonia, pulmonary embolism, respiratory depression, seizures, and swollen knee; or complications relating to deep infection. Adverse events were vomiting and nausea.

\section{Study quality}

Potential sources of bias were recorded in a Cochrane risk of bias table [14]. We considered random sequence generation, allocation concealment, blinding of participants and personnel, blind outcome assessment, incomplete outcome data, selective reporting, and other sources of bias. We classified overall quality as low, unclear or high risk of bias.

\section{Meta-analysis}

We conducted meta-analyses for pain at rest and during activity at 24 and 48 hours, length of hospital stay, and 
Table 1 Search strategy as applied in MEDLINE

1 Anesthetics, Local/or local anaesthetic.mp.

2 Anesthetics, Local/or Anesthesia, Local/or Local anaesthesia.mp.

3 Anesthetics/or Anesthesia/or anaesthesia.mp. or Anesthetics, Local/or Anesthesia, Local/

4 anesthesia.mp.

5 anaesthetic.mp

6 amides.mp. or Amides/

7 ("Huneke neural therapy" or "Neural therapy of Huneke" or benzocaine or bensokain or "Aminobenzoic Acid" or "Aminobenzoate" or bupivacain* or buvacaina or sensorcaine or marcain* or svedocain* or levobupivacaine or carticain* or articain* or dibucaine or cinchocaine or Cincain or Nupercain* or Sovcaine or etidocaine or duranest or "W19053" or "W 19053" or "W-19053" or Lidocaine or Lignocaine or Octocaine or Xylesthesin or Xylocaine or Dalcaine or Xylocitin or Xyloneural or Mepivacain* or Carbocaine or Polocaine or isocaine or isogaine or Scandicain* or prilocaine or Propitocaine or Tetracaine or Tetrakain or Amethocaine or Dicaine or Pantocaine or Pontocaine or Trimecaine or Mesocaine or ropivacaine).mp. $[\mathrm{mp}=$ title, abstract, original title, name of substance word, subject heading word, protocol supplementary concept, rare disease supplementary concept, unique identifier]

81 or 2 or 3 or 4 or 5 or 6 or 7

9 (incision or port* or (surg* and wound)).mp. [mp = title, abstract, original title, name of substance word, subject heading word, protocol supplementary concept, rare disease supplementary concept, unique identifier]

10 acetabular.mp.

11 infiltration.mp.

12 wound infiltration.mp.

13 wound catheter.mp.

14 peri-articular.mp.

15 periarticular.mp.

16 intraarticular.mp

17 intra-articular.mp

189 or 10 or 11 or 12 or 13 or 14 or 15 or 16 or 17

19 Arthroplasty, Replacement, Knee/or Arthroplasty, Replacement, Hip/

20 exp Arthroplasty, Replacement, Hip/or exp Hip Prosthesis/ or hip replacement.mp.

21 exp Arthroplasty, Replacement, Knee/or exp Knee Prosthesis/or knee replacement.mp.

22 knee prosthesis.mp. or exp Knee Prosthesis/

23 hip prosthesis.mp. or exp Hip Prosthesis/

24 total hip.tw.

25 total knee.tw.

26 Orthopedic Procedures/ or orthopaedic surgery.mp.

2719 or 20 or 21 or 22 or 23 or 24 or 25 or 26

$28 \quad 8$ and 18 and 27

*Ovid truncation character.

complications. Data collected on mobilisation, long-term pain and function outcomes were not suitable for metaanalyses and results were summarised using a descriptive narrative.

Follow-up times were approximated to the closest timing. When not specified, we assumed measurements were taken at rest. Analyses were carried out in Stata 12 and Review Manager 5 and results are reported with 95\% confidence intervals. Funnel plots were inspected to assess small study effects [20]. Given the number of potential effect modifiers, we used random effects models for all meta-analyses.
In meta-analysis, means and standard deviations of continuous variables such as pain intensity are required for intervention and control groups. Pain outcomes are sometimes reported as medians and inter-quartile ranges due to the recognised ceiling effects of pain measures after successful pain management. However this is less of an issue during early recovery. Kerr and Kohan presented distributions of pain intensity scores at rest and during walking on the first and second day after THR or TKR [19]. The proportion of people reporting no pain, and thus reflecting a ceiling effect, ranged from 2 to $35 \%$ on days one and two and pain intensities showed near normal distributions. 
Assuming a normal distribution, we estimated means and standard deviations from medians and inter-quartile ranges [14]. If no measures of variability were available in articles, we contacted authors to obtain standard deviations. If unavailable we used the method described by Walter and Yao to estimate standard deviations from ranges [21], or imputed values from the average across studies with the same outcome.

As pain scores are reported on different scales we used the standardised mean difference (SMD) as our measure of treatment effect in meta-analyses [22]. To help in the interpretation of the pooled estimates, we multiplied SMD values by the mean standard deviation on the widely reported 100 point VAS scale for the outcome. As the use of this method is entirely dependent on the chosen "typical" value [23], we used a mean standard deviation calculated from all studies reporting the outcome [14].

For length of hospital stay, we compared means and medians in studies reporting both, and examined individual level data provided by some authors. Distributions were right-skewed and followed a lognormal distribution. Some studies reported means and standard deviations directly. For studies that reported medians and inter-quartile ranges, or ranges, we estimated means and standard deviations on the log scale and then backtransformed them to the natural (unlogged) scale [24]. We reported the mean difference (MD) in days as our measure of treatment effect in meta-analyses. Complications were compared between randomised groups using meta-analysis with summary statistics calculated as the Peto odds ratio (OR), the method of choice when event rates are low $[14,25]$.

\section{Analgesia regimen comparisons}

Not all studies compared a local anaesthetic infiltration intervention with no intervention or placebo. Thus meta-analyses are reported separately for different regimen comparisons, which we label A-E. These are summarised in Figure 1.

THR studies were grouped into comparisons A and B; we and also report the combined comparison $(A+B)$.

For TKR studies, we initially report results from a combined meta-analysis across the first two subgroups $(A+B)$, comparing local anaesthetic infiltration with or without further post-closure intervention against control. Further analyses report the comparisons C, D and E.

\section{Heterogeneity and subgroup analyses}

We quantified the differences in treatment effects between groups using meta-regression. Heterogeneity within metaanalyses was quantified using the $\mathrm{\tau}^{2}$ and $\mathrm{I}^{2}$ statistics [26]. Sensitivity and sub-group analyses explored risk of bias in the study, use of additional analgesia delivered through a

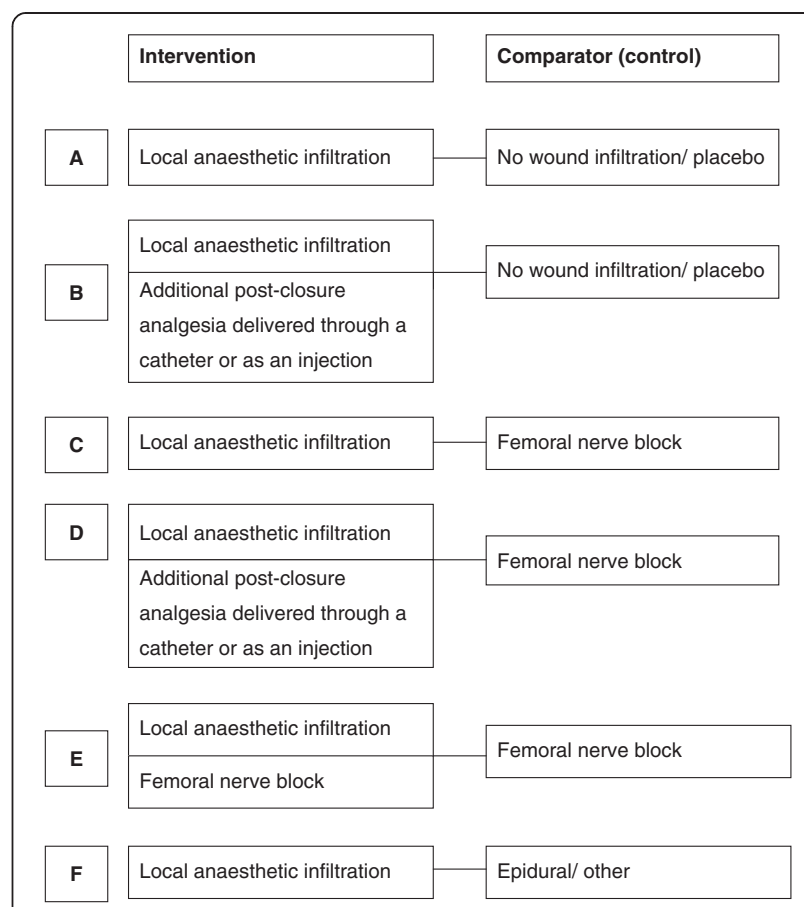

Figure 1 Wound infiltration anaesthesia regimens for interventions and comparators (controls).

catheter or injection, and inclusion of non-steroidal inflammatory agents or steroids in the infiltrate.

\section{Results}

Searches identified 839 articles of which 33 described 36 RCTs evaluating local anaesthetic infiltration in THR or TKR. The flow diagram in Figure 2 summarises review progress. Some consistency in outcome reporting was apparent for pain outcomes but for opioid consumption and ambulation the variety of outcomes precluded metaanalysis.

\section{Small study effects}

Inspection of funnel plots for each meta-analysis gave no strong indication of publication bias or small study effects, but numbers of studies in individual analysis groups were small such that it was difficult to assess asymmetry.

\section{Total hip replacement}

Details of 13 studies including 909 patients with THR [16,27-37], or a large majority with THR [10], are summarised in Table 2 which also includes our summary risk of bias assessment. A more detailed assessment of risk of bias is included in Additional file 2. The mean number of patients randomised was 70 (range 37-120). We assessed that 10 studies were at low risk of bias while three studies had unclear risk of bias due to uncertainty about blinding of outcome assessments. 


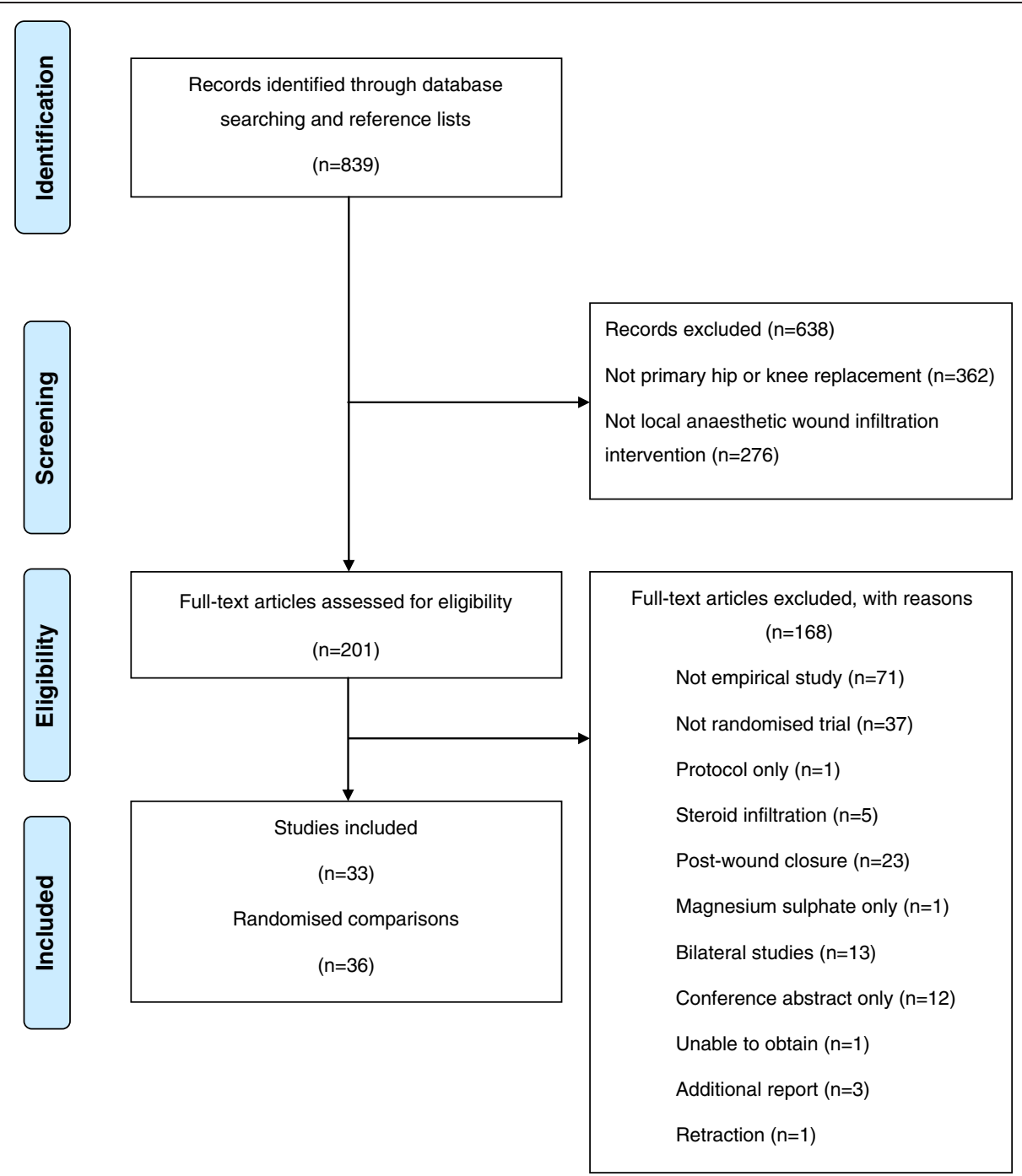

Figure 2 Systematic review flow diagram.

\section{Pain}

Results of meta-analyses including up to 12 studies [10,16,27-29,31-37], are summarised in Table 3 and Figure 3. In patients receiving local anaesthetic infiltration (A and $\mathrm{B}$ ), there was strong evidence that pain was lower: at rest at 24 hours by SMD -0.61 ( $95 \%$ CI $-1.05,-0.16 ; \mathrm{p}=0.008$ ), and during activity by SMD -0.85 (95\% CI $-1.45,-0.25 ; \mathrm{p}=$ 0.006). This reflected reduced pain at 24 hours at rest by an average of 12 points (95\% CI 3, 21; $\mathrm{p}=0.008)$, and during activity by 24 points $(95 \%$ CI 7,$42 ; \mathrm{p}=0.006)$ on a 100 point scale. Average effect sizes at 48 hours were smaller for pain at rest, SMD $-0.29(95 \% \mathrm{CI}-0.52,-0.05 ; \mathrm{p}=0.018)$ and during activity, SMD -0.43 (95\% CI $-0.78,-0.09 ; \mathrm{p}=0.014)$, corresponding to 5 and 10 points on a 100 point scale, respectively.

In seven studies with no additional post-closure analgesia through a catheter or injection $(\mathrm{A})$, patients receiving local anaesthetic infiltration reported lower pain at 24 hours at rest by SMD $-0.63(95 \% \mathrm{CI}-1.21,-0.06 ; \mathrm{p}=0.031)$, equivalent to an average of 12 points lower pain. However, there was no strong evidence that the intervention had an effect during activity or at 48 hours.

In five studies where patients received further post-closure analgesia (B), pain was reduced on average at 24 hours during activity by SMD -1.38 (95\% CI $-2.5,-0.26 ; \mathrm{p}=0.016)$, equivalent to a 40 point decrease on a 100 point scale. Pain at 48 hours was reduced, on average, at rest by SMD -0.49 (95\% CI, $-0.96,-0.02 ; \mathrm{p}=0.043)$ and during activity by SMD $-0.6(95 \% \mathrm{CI}-1.16,-0.04 ; \mathrm{p}=0.036)$ equivalent to 8 and 14 point decreases, respectively.

In one study, control patients received an epidural analgesia infusion [16]. Pain was lower for the duration of the epidural infusion, but at 48 hours pain was higher in the control group compared with the local anaesthetic 
Table 2 Randomised controlled trials of local anaesthetic infiltration in total knee and hip replacement

\begin{tabular}{llll}
\hline Study country date & $\begin{array}{l}\text { Inclusion patients } \\
\text { (intervention: control) }\end{array}$ & $\begin{array}{l}\text { Common treatment } \\
\text { Intervention treatment (infiltrate volume) } \\
\text { Further treatment (if given) }\end{array}$ & $\begin{array}{l}\text { Latest post-surgical follow up Outcomes } \\
\text { Losses to follow up (intervention/ control) } \\
\text { Risk of bias summary }\end{array}$ \\
\hline
\end{tabular}

\section{TOTAL HIP REPLACEMENT STUDIES}

Aguirre et al. 2012 [34]

Switzerland Not specified

THR (minimally invasive) $N=76$

(38:38) $58: 58$ years $53: 50 \%$

female

Andersen KV et al. 2007 [16] THR, OA, elective N = 80 (40:40) Denmark 2005-2006 62:61 years 90:85\% female

Andersen L et al. 2007 [27] Denmark Date not specified

THR, OA, uncemented, $>80$ years $N=37(19: 18) 62: 64$ years $84: 56 \%$ female

Bianconi et al. 2003 [10] Italy Date not specified

Busch et al. 2010 [30] UK 2003-2005

Dobie et al. 2012 [35] UK 2006-2007

THR OA or RA N $=96$ (50:46)

67:67 years $38: 52 \%$ female 2006-2007 Note: additio and epidural

THR and TKR (78\% THR), elective $N=37(18: 19) 66: 64$ years $79: 83 \%$ female

THR, $\mathrm{OA}$, age $<80$ years $\mathrm{N}=64$ (32:32) $61: 65$ years $50: 54 \%$ female female
Spinal anaesthesia, PCA morphine

$20 \mathrm{ml}$ solution containing $60 \mathrm{mg}$ ropivacaine injected into wound before closure. Further continuous infusion through catheter

Spinal, post-operative oral oxycodon hydrochloride as required

$101.5 \mathrm{ml}$ solution containing $200 \mathrm{mg}$ ropivacaine, $30 \mathrm{mg}$ ketoralac and $0.5 \mathrm{mg}$ epinephrine infiltrated during surgery. Further infiltrate through catheter intra-articularly 8 hours after surgery.

Spinal anaesthesia, self-administered oral oxycodone as rescue medication

$151.5 \mathrm{ml}$ saline solution containing $300 \mathrm{mg}$ ropivacaine, $30 \mathrm{mg}$ ketorolac, and $0.5 \mathrm{mg}$ adrenaline infiltrated during surgery Further infusion through catheter on day 1 .

THR, 13\% OA, 72\% Osteonecrosis $\mathrm{N}=60(30: 30) 51: 55$ years $37: 43 \%$
Spinal anaesthesia. Loading dose of intravenous morphine at end of surgery

$40 \mathrm{ml}$ saline containing $200 \mathrm{mg}$ ropivacaine infiltrated at end of surgery. Further ropivacaine infusion through catheter for 55 hours after closure Intravenous saline infusion for $24 \mathrm{~h}$ after surgery.

General or spinal anaesthesia, PCA morphine

$100 \mathrm{ml}$ saline solution containing $400 \mathrm{mg}$ ropivacaine, $30 \mathrm{mg}$ ketorolac, $5 \mathrm{mg}$ epimorphine, and $0.6 \mathrm{ml}$ epinephrine (1:1000) infiltrated during surgery.

Spinal, general, intravenous morphine after surgery as required.

$160 \mathrm{ml}$ saline solution containing $200 \mathrm{mg}$ levobupivacaine and adrenaline

\section{General anaesthesia}

Pre-emptive analgesia with oral Oxycodone and Celecoxib. Epidural anaesthesia. $90 \mathrm{ml}$ saline
$20 \mathrm{ml}$ placebo injection of saline Continuous infusion of saline through cathete

Epidural infusion of ropivacaine

and morphine

Saline placebo infiltration Saline
placebo infused through
catheter on day 1

6 weeks VAS pain at rest and 6 8 hours, WOMAC pain to day 4, WOMAC pain, stiffness and function after 1,2,4,6 weeks, EQ5D 6 weeks, patient controlled analgesic use to discharge, adverse events 3 patients out of 10 not fitting inclusion criteria were identified retrospectively. No losses to follow up Low risk of bias

72 hours VAS pain at 2,4,8,12,24,48,72 hours, opioid consumption (rescue medication).

No placebo infiltration during surgery. Saline infusion through catheter for $55 \mathrm{~h}$ after closure. Intravenous morphine plus ketorolac infusion ff adverse events, length of hospital stay, patient satisfaction No losses to follow up Low risk of bias

2 years VAS at rest and activity, morphine consumption (PCA), VAS satisfaction,

No placebo infiltration complications, Harris Hip Score, WOMAC length of hospital stay No losses to follow up Low risk of bias

6 days VAS at $24 \mathrm{~h}$, morphine consumption, walking and stair test, mobilisation velocity and day, sit to stand test, home readiness, hospital stay, lowa Level of Assistance Scale $4(4: 0)$ patients did not receive intervention as planned. Intention to treat results. Some data missing for 1 control Low risk of bias

5 days VAS pain, ambulation, doses of No pre-emptive analgesia No parenteral analgesia, time to straight leg raise, epidural No injection during surgery complications No losses to follow up described

Unclear (blinding of outcome assessment) 
Table 2 Randomised controlled trials of local anaesthetic infiltration in total knee and hip replacement (Continued)

\begin{tabular}{|c|c|c|}
\hline & & $\begin{array}{l}\text { solution containing } 5 \mathrm{mg} \text { morphine, } 40 \mathrm{mg} \\
\text { methylprednisolone and } 6.8 \mathrm{mg} \text { ropivacaine } \\
\text { infiltrated during surgery. Post-operative oral } \\
\text { Oxycodone and paracetamol. }\end{array}$ \\
\hline \multirow{2}{*}{$\begin{array}{l}\text { Liu et al. } 2011 \text { [32] China } \\
\text { 2008-2009 }\end{array}$} & \multirow{2}{*}{$\begin{array}{l}\text { THR, OA, ASA I-III, }<80 \text { years } \\
N=82(41: 41) 74: 74 \text { years } \\
75: 77 \% \text { female }\end{array}$} & Spinal anaesthesia, PCA morphine \\
\hline & & $\begin{array}{l}60 \mathrm{ml} \text { saline solution containing } 5 \mathrm{mg} \text { morphine, } \\
30 \mathrm{mg} \text { bupivacaine, } 1 \mathrm{ml} \text { betamethasone and } \\
0.5 \mathrm{ml} \text { epinephrine infiltrated during surgery. }\end{array}$ \\
\hline \multirow[t]{2}{*}{$\begin{array}{l}\text { Lu et al. } 2010 \text { [31] China Not } \\
\text { specified }\end{array}$} & \multirow{2}{*}{$\begin{array}{l}\text { THR, primary } N=40(20: 20) \text { No } \\
\text { information on age and sex of } \\
\text { patients }\end{array}$} & $\begin{array}{l}\text { No description of common anaesthesia except } \\
\text { PCA }\end{array}$ \\
\hline & & $\begin{array}{l}\text { COX-2 inhibitor before surgery. } 100 \mathrm{ml} \text { solution } \\
\text { containing } 0.15 \% \text { ropivacaine infiltrated at end of } \\
\text { surgery. COX- } 2 \text { inhibitor after surgery }\end{array}$ \\
\hline \multirow[t]{2}{*}{$\begin{array}{l}\text { Lunn et al. } 2011 \text { [33] Denmark } \\
\text { 2009-2010 }\end{array}$} & \multirow[t]{2}{*}{$\begin{array}{l}\text { THR, }>18 \text { years } N=120(60: 60) \\
67: 67 \text { years } 55: 65 \% \text { female }\end{array}$} & $\begin{array}{l}\text { Spinal with or without general. Multimodal oral } \\
\text { analgesia }\end{array}$ \\
\hline & & $\begin{array}{l}150 \mathrm{ml} \text { saline solution containing } 0.2 \% \\
\text { ropivacaine and } 10 \mu \mathrm{g} / \mathrm{ml} \text { epinephrine } \\
\text { infiltrated during surgery. }\end{array}$ \\
\hline \multirow{2}{*}{$\begin{array}{l}\text { Murphy et al. } 2012 \text { [36] Ireland } \\
\text { 2009-2010 }\end{array}$} & \multirow{2}{*}{$\begin{array}{l}\text { THR, } \mathrm{OAN}=91(45: 46) \\
57: 54 \text { years } 49: 38 \% \text { female }\end{array}$} & Spinal, PCA opioid analgesia \\
\hline & & $\begin{array}{l}60 \mathrm{ml} \text { saline containing } 150 \mathrm{mg} \text { levobupivacaine } \\
\text { infiltrated during surgery. }\end{array}$ \\
\hline \multirow{2}{*}{$\begin{array}{l}\text { Parvataneni et al. Hip } 2007 \\
\text { [28] USA 2005-2006 }\end{array}$} & \multirow{2}{*}{$\begin{array}{l}\text { THR, } O A N=71(35: 36) \\
64: 61 \text { years } 40: 39 \% \text { female }\end{array}$} & Spinal anaesthesia with or without FNB \\
\hline & & $\begin{array}{l}\text { Intra-operative infiltration of } 200-400 \mathrm{mg} \\
\text { bupivacaine, } 4-10 \mathrm{mg} \text { morphine sulphate } \\
300 \mathrm{\mu g} \text { epinephrine, } 40 \mathrm{mg} \text { methylprednisolone } \\
\text { acetate, } 75 \mathrm{mg} \text { cefuroxime and } 22 \mathrm{ml} \text { saline. } \\
\text { Total volume approximately } 33 \mathrm{ml} \text {. }\end{array}$ \\
\hline \multirow[t]{2}{*}{$\begin{array}{l}\text { Rikalainen-Salmi et al. } 2012 \\
\text { [37] Finland 2009-2010 }\end{array}$} & \multirow{2}{*}{$\begin{array}{l}\text { THR, OA, ASA I-III N }=60(30: 30) \\
65: 66 \text { years (followed up) } 66: 61 \% \\
\text { female (followed up) }\end{array}$} & $\begin{array}{l}\text { Spinal, propofol if required, oxycodone rescue } \\
\text { medication. }\end{array}$ \\
\hline & & $\begin{array}{l}101 \mathrm{ml} \text { solution containing } 125 \mathrm{mg} \\
\text { levobupivacaine, } 30 \mathrm{mg} \text { ketorolac infiltrated } \\
\text { during surgery } 21 \mathrm{ml} \text { solution containing } \\
100 \mathrm{mg} \text { levobupivacaine and } 30 \mathrm{mg} \text { ketoralac } \\
\text { administered through catheter on morning of } \\
\text { first post-operative day }\end{array}$ \\
\hline \multicolumn{3}{|c|}{ TOTAL KNEE REPLACEMENT STUDIES } \\
\hline \multirow{2}{*}{$\begin{array}{l}\text { Affas et al. } 2011 \text { [50] Sweden } \\
\text { 2007-2008 }\end{array}$} & \multirow{2}{*}{$\begin{array}{l}\text { TKR, } 77.5 \% \text { OA, } 22.5 \% \text { RA, }>18 \text { years, } \\
\text { ASA I-III, primary. } \\
\mathrm{N}=40 \text { (20:20) } 67: 69 \text { years } 45: 60 \% \\
\text { female }\end{array}$} & Spinal anaesthesia, PCA morphine \\
\hline & & $\begin{array}{l}110 \mathrm{ml} \text { containing approximately } 200 \mathrm{mg} \\
\text { ropivacaine, } 20 \mathrm{mg} \text { ketorolac and } 0.33 \mathrm{mg} \\
\text { epinephrine infiltrated during surgery. Further } \\
\text { intra-articular infiltration through catheter after } \\
\text { surgery. }\end{array}$ \\
\hline
\end{tabular}

Post-operative intravenous PCA

and oral and injected analgesics

as required

$60 \mathrm{ml}$ saline infiltrated during surgery.

15 days and 9 months (range $6-12$ months) for infection Morphine use, VAS pain, surgical outcome, mobilisation (time to straight leg raise and 90 degree flexion) 2 (1:1) lost to follow up Low risk of bias

48 hours VAS pain, use of PCA pump, adverse drug reactions No losses to follow up apparent

No COX-2 inhibitor before Unclear (limited reporting)

surgery $100 \mathrm{ml}$ saline placebo infiltrated at end of surgery. No COX-2 inhibitor after surgery

8 hours and to discharge VAS pain at rest and during walking and passive hip flexion, Oxycodone

$150 \mathrm{ml}$ saline placebo infiltrated consumption, complications No losses to follow up

during surgery.

except "pain during walking" with 18 (11:7) lost to

follow up Low risk of bias (except pain during activity: possible risk of bias due to large number of patients unable to complete test)

72 hours WOMAC Pain, McGill Pain Questionnaire, VAS pain, morphine consumption, complications 13 (6:7) lost to follow up but some analyses used multi-level modelling to handle missing data Low risk of bias

3 months VAS pain, total narcotic dose, functional recovery including time to straight leg raise, side

No infiltration during surgery Post surgical PCA effects of narcotic us losses to follow up reported Low risk of bias

8 weeks NRS pain at rest and motion, oxycodone consumption, mobilisation, fulfilment of discharge criteria, satisfaction, adverse events and

Intrathecal morphine No placebo infiltration Sham catheter attached to skin with $21 \mathrm{ml}$ air administered on morning of first post-operative day (not inserted into joint)

24 hours NRS pain intensity at rest and on movement, 24 hour morphine PCA consumption. Femoral nerve block. Intravenous No losses to follow up. Missing data analysis ketorolac after surgery. No placebo infiltration. reported. Unclear risk of bias (blinding of outcome assessment) 
$67: 69$ years $43: 26 \%$ female

Busch et al. 2006 [38] Canada Date not specified

$\mathrm{KR}$, age $<80$ years $\mathrm{N}=64$ 32:32) $66: 70$ years 50:59\% female

Carli et al. 2010 [45] Canada 2007-2008

TKR, OA, tricompartmental cemented. $\mathrm{N}=40(20 \cdot 20)$

Chen et al. 2012 [52] China 2008

Essving et al. 2010 [46] Sweden 2007-2008

Essving et al. 2011 [51] Sweden 2009-2010

Fu et al. 2009 [42] China 2006-2007

Fu et al. 2010 [47] China 2008-2009 (40:41) $66: 65$ years $75: 78 \%$ female female 64:60\% female $69: 68$ years $75: 78 \%$ female (50:50) 68:67 years $76: 80 \%$
Spinal anaesthesia, PCA morphine

$151.5 \mathrm{ml}$ saline solution containing $300 \mathrm{mg}$ ropivacaine, $30 \mathrm{mg}$ ketorolac and $0.5 \mathrm{mg}$ epinephrine infiltrated during surgery. Further continuous infusion through catheter after closure.

General or spinal anaesthesia, PCA morphine

$100 \mathrm{ml}$ saline solution containing $400 \mathrm{mg}$

ropivacaine, $30 \mathrm{mg}$ ketorolac, $5 \mathrm{mg}$

epimorphine, and $0.6 \mathrm{ml}$ epinephrine $(1: 1000)$ infiltrated during surgery.

Spinal anaesthesia, PCA morphine

Solution of ropivacaine $(0.2 \%), 1 \mathrm{ml}$ of ketorolac $(30 \mathrm{mg} / \mathrm{ml})$, and $0.5 \mathrm{ml}$ of epinephrine $(1 \mathrm{mg} / \mathrm{ml})$ with a total volume of $100 \mathrm{ml}$ infiltrated during surgery. Further infusion through catheter after closure

TKR, $\mathrm{OA}$, age $<76$ years. $\mathrm{N}=$

\section{Spinal anaesthesia, PCA morphine}

Intra-operative injection of a solution of magnesium sulphate $(50 \mathrm{mg} / \mathrm{kg})$ and $190 \mathrm{mg}$ ropivacaine in normal saline to a volume of $100 \mathrm{ml}$

TKR, OA, ASA I-III, 20-85 years $N=48(24: 24) 72.70$ years $54: 54 \%$

TKR, OA, ASA I-III, age 40-85 years $N=50(25: 25) 71: 71$ years

TKR, OA, age $<80 y \mathrm{~N}=80(40: 40)$

TKR, OA, age $<80$ years $N=100$

female

General anaesthesia, PCA morphine

$116 \mathrm{ml}$ saline containing $300 \mathrm{mg}$ ropivacaine $30 \mathrm{mg}$ ketorolac and $0.5 \mathrm{mg}$ epinephrine infiltrated during surgery. $50 \mathrm{ml}$ saline containing $100 \mathrm{mg}$ ropivacaine infiltrated before closure. Further injection of mixture $21 \mathrm{~h}$ after closure.

Spinal anaesthesia, PCA morphine

Spinal plus intrathecal saline. Injection during surgery of $400 \mathrm{mg}$ ropivacaine $(160 \mathrm{ml}), 30 \mathrm{mg}$ ketoralac $(1 \mathrm{ml})$ and $0.5 \mathrm{mg}$ epinephrine $(5 \mathrm{ml})$ Further infiltrate through catheter on day and 2

Spinal anaesthesia, PCA morphine

$60 \mathrm{ml}$ saline containing $5 \mathrm{mg}$ morphine, $30 \mathrm{mg}$ bupivacaine and $1 \mathrm{ml}$ betamethasone infiltrated during surgery.

Spinal anaesthesia, PCA morphine

Oral COX-2 inhibitor and tramadol 1 day before
Epidural infusion of ropivacaine Post-operative intravenous ketorolac

No placebo infiltration to 1 month after surgery $50 \mathrm{ml}$ saline containin $5 \mathrm{mg}$ morphine, $150 \mathrm{mg}$ ropivacaine, $0.5 \mathrm{ml}$ adrenaline and $1 \mathrm{ml}$ betamethasone infiltrated during surgery.

Continuous femoral nerve block infusion of saline niection of $100 \mathrm{~m} /$ norma saline

No placebo injections during surgery. Post-surgical injection of saline at 21 hours.

Spinal plus intrathecal morphin No injection during surgery al infusion of saline through catheter

$60 \mathrm{ml}$ saline infiltrated during surgery

Oral placebo 1 day before to saline placebo infiltrated during surgery
72 hours and to discharge. Infection to 30 days VAS/ NRS pain, morphine requirement, side effects and complications, time to achieve discharge criteria, ength of stay 9 (3.6) patients lost to follow up Unclear risk of bias (blinding of outcome assessment)

6 weeks VAS at rest and activity, morphine consumption (PCA), VAS satisfaction, complications, Knee Society Score, WOMAC, length of hospital stay No losses to follow up Low risk of bias

6 weeks Morphine consumption, NRS pain at rest and walking, functional capacity, ability to walk Saline injection Post-surgical

ch-12, WOMAC No follow up Low risk of bias

15 days and infection to 6 months Total morphine consumption, VAS pain at rest and motion, time to straight leg raise and 90 degree flexion, adverse events including delayed infection 1 (0:1) patient lost to follow up Low risk of bias

3 months PCA morphine consumption, VAS pain rest and on knee flexion, time to home readiness, ength of hospital stay, surgical outcome, function eutcome tests, Oxford Knee Score, EQ-5D, patient satisfaction, adverse events $1(0: 1)$ patient lost to follow up Low risk of bias

3 months VAS pain, PCA morphine, verbal rating scale of satisfaction, functional tests, time to home $2(0: 2)$ patients lost to follow up Low risk of bias month after surgery $50 \mathrm{ml}$
15 days except ROM 90 days, infection 12 months Morphine consumption, VAS pain at rest and activity, ROM, time to straight leg raise, surgical outcomes, complications. No losses to follow up. Missing data imputation described Low risk of bias

15 days except ROM at 90 days and infection to morphine consumption (PCA and intramuscular), time to straight leg raise and 90 degree flexion, surgical outcomes, adverse reactions No losses to follow up Low risk of bias mean 7.5 months (range 6-9 months) VAS pain 


\section{Han et al. 20071 and 2 [40] TKR, primary $N=(30: 30: 30)$}

Korea 2005-2006 Note: 2 intervention groups

69:68:67 years 90:80:90\% female

Spinal and epidural anaesthesia, PCA morphine

1) $50 \mathrm{ml}$ saline solution containing $300 \mathrm{mg}$ ropivacaine, epinephrine $(0.25 \mathrm{ml} 1: 200,000)$ and $5 \mathrm{mg}$ morphine injected before wound closure. 2) $50 \mathrm{ml}$ saline solution containing $300 \mathrm{mg}$ ropivacaine and epinephrine $(0.25 \mathrm{ml} 1: 200,000)$ injected before wound closure.

Koh et al. 2012 [53] Kore 2008-2009

TKR, OA, unilateral $\mathrm{N}=10$ (49:52) $70: 70$ years $89: 91 \%$ female

FNB, spinal anaesthesia, PCA morphine

$50 \mathrm{ml}$ saline containing ropivacaine $300 \mathrm{mg}$ morphine sulphate $10 \mathrm{mg}$, ketoralac $30 \mathrm{mg}$, $0.3 \mathrm{mg}$ epinephrine, cefuroxime $750 \mathrm{mg}$ injected/ infiltrated during surgery.

Krenzel et al. 2009 [43] USA 2007-2008

Mahadevan et al. 2012 [54] UK Not specified

Meftah et al. 2012 [55] USA 2010-2011

$\mathrm{Ng}$ et al. 2012 [56] China 2008-2010 Note: crossover design. Patients having both knees replaced

Parvataneni et al. Knee 2007 [28] USA 2005-2006

Spreng et al. no iv injection 2010[48] Norway 2007-2009

TKR, unilateral, non-cemented, no patella resurfacing, age $>17$ years, ASA I-III. $\mathrm{N}=68$ (34:34) 67:66 years 61:67\% female
TKR, 96\% OA elective. $\mathrm{N}=67$ (35:32), 1 patient with staged bilateral TKR included twice. $67: 65$ years $57: 72 \%$ female

TKR, OA or RA, unilateral $\mathrm{N}=52$ (26:26) $68: 67$ years $54: 58 \%$ female

TKR, unilateral $\mathrm{N}=90$ (45:45) 65:67 years $64: 64 \%$ female

TKR, OA N = 32 (16:16) surgeries but 16 patients only having 2 TKRs 3 months apart $70 \cdot 70$ years 88:88\% female

TKR, OA N = 60 (31:29) $69: 71$ years $45: 52 \%$ female
FNB, spinal anaesthesia, PCA fentanyl

$20 \mathrm{ml}$ infiltration of $100 \mathrm{mg}$ ropivacaine during surgery.

FNB, general anaesthesia, PCA morphine.

$25 \mathrm{ml}$ saline containing $0.375 \%$ levobupivacaine infiltrated during surgery.

Pre-emptive analgesia

$45.1 \mathrm{ml}$ saline solution containing marcaine (400-800 mg, morphine sulphate 8 mg adrenaline $0.3 \mathrm{mg}$ antibiotic $750 \mathrm{mg}$ corticosteroids $40 \mathrm{mg}$ injected during surgery.

General anaesthesia, remifentanil infusion PCA morphine

$101.5 \mathrm{ml}$ saline solution containing $300 \mathrm{mg}$ ropivacaine, adrenaline $1 \mathrm{mg}$ and triamcinolone acetonide $40 \mathrm{mg}$ infiltrated during surgery. Femoral catheter inserted and saline infused.

Spinal anaesthesia with or without FNB Intra-operative infiltration of 200-400 mg bupivacaine, 4-10 mg morphine sulphate $300 \mathrm{\mu g}$ epinephrine, $40 \mathrm{mg}$ methylprednisolone acetate, $75 \mathrm{mg}$ cefuroxime and $22 \mathrm{ml}$ saline. Total volume approximately $33 \mathrm{ml}$.

Spinal. Propofol if indicated. PCA morphine

$150 \mathrm{ml}$ saline solution containing $150 \mathrm{mg}$ ropivacaine, $0.5 \mathrm{mg}$ epinephrine, $30 \mathrm{mg}$

ketorolac and $5 \mathrm{mg}$ morphine infiltrated during surgery. Knee injected through catheter with ropivacaine and ketorolac solution after 22-24 hours Intravenous injection with saline at 22-24 hours

48 hours Incidence of booster PCA for 24 hours, amount of intra-venous tramadol VAS pain at rest and exercising, side effects, range of flexion. No losses to follow up reported Low risk of bias

$50 \mathrm{ml}$ saline placebo

No placebo infiltration reported

$20 \mathrm{ml}$ saline placebo infiltrated during surgery

Sciatic nerve block No placebo infiltration reported

FNB. PCA epidural No placebo injection reported

Femoral nerve block. Wound infiltration with $101.5 \mathrm{ml}$ salin

No infiltration during surgery Femoral nerve block at end of surgery Post surgical PCA Effor to conceal allocation but no sham epidural

48 hours of epidural analgesia as soon as spinal started to $w$ off No wound infiltration during surgery. No injections through surgery. No injections throu
sham catheter. No sham epidurals
7 days VAS pain at rest (day 1) and on movement (days 4 and 7), PCA opioid consumption, use of rescue medication, pain compared with expectations, functional recovery (straight leg raise and flexion), satisfaction, side-effects and complications, length of stay. 14 (4:10) did not receive treatment as planned. Results reported by intention to treat Low risk of bias

24 hours PCA fentanyl consumption, NRS pain, functional tests, time to straight leg raise, ambulation distance, surgical outcomes, adverse events No losses to follow up Low risk of bias

48 hours and to discharge VAS pain, morphine consumption, active ROM, length of hospital stay. No losses to follow up reported Low risk of bias

3 days and to discharge. 6 months for infection, fracture and re-operation. Pain at rest and ambulation, readiness for discharge.1 (1:0) lost to all follow up, 6 (4:2) lost to readiness for discharge follow up Unclear (blinding of outcome assessment)

3 days and to discharge Pain score at rest and motion, total morphine consumption, Knee Society Score, ROM, quadriceps power, satisfaction, adverse events and complications. No losses to follow up reported Low risk of bias

3 months VAS pain, total narcotic dose, functional recovery including time to straight leg raise, side effects of narcotic use, patient satisfaction No losses to follow up reported Low risk of bias

72 hours and to discharge VAS at rest and during knee flexion, morphine consumption, functional recovery, length of stay, satisfaction, mobilisation including walking distance, adverse events2 (1:1) lost to follow up Low risk of bias 
Table 2 Randomised controlled trials of local anaesthetic infiltration in total knee and hip replacement (Continued)

\begin{tabular}{|c|c|c|c|}
\hline \multirow[t]{2}{*}{$\begin{array}{l}\text { Spreng et al. with iv injection } \\
2010 \text { [48] Norway 2007-2009 }\end{array}$} & \multirow{2}{*}{$\begin{array}{l}\text { TKR, unilateral, non-cemented, } \\
\text { no patella resurfacing, age } \\
\text { >17 years, ASA I-III. } N=68 \\
\text { (34:34) 67:66 years } 61: 67 \% \\
\text { female }\end{array}$} & \multicolumn{2}{|l|}{$\begin{array}{l}\text { Spinal anaesthesia, propofol if indicated, PCA } \\
\text { morphine }\end{array}$} \\
\hline & & 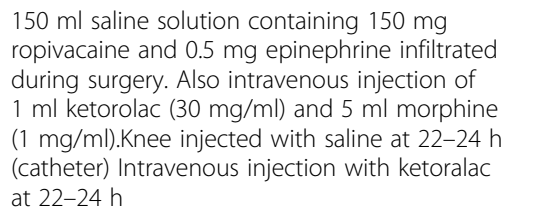 & $\begin{array}{l}48 \text { hours of epidural analgesia } \\
\text { as soon as spinal anaesthetic } \\
\text { started to wear off. No wound } \\
\text { infiltration during surgery. No } \\
\text { injections through sham catheter }\end{array}$ \\
\hline \multirow{2}{*}{$\begin{array}{l}\text { Thorsell et al. } 2010 \text { [49] Sweden } \\
\text { Not specified }\end{array}$} & \multirow{2}{*}{$\begin{array}{l}\text { TKR, OA or RA N }=85 \text { ( } 46: 39) \\
69: 72 \text { years (followed up) } 81: 73 \% \\
\text { female (followed up) }\end{array}$} & Not specified, probable PCA & \\
\hline & & $\begin{array}{l}\text { Spinal anaesthesia } 156 \mathrm{ml} \text { solution with } 300 \mathrm{mg} \\
\text { ropivacaine, } 0.5 \mathrm{mg} \text { adrenaline and } 30 \mathrm{mg} \\
\text { ketorolac infiltrated during surgery. Further } \\
\text { infiltrate through catheter intra-articularly on } \\
\text { post-operative day } 1 .\end{array}$ & $\begin{array}{l}\text { Spinal or epidural analgesia No } \\
\text { placebo infiltration reported } \\
\text { Post-operative pain relief with } \\
\text { ropivacaine infusion through } \\
\text { epidural catheter. }\end{array}$ \\
\hline \multirow[t]{2}{*}{$\begin{array}{l}\text { Toftdahl et al. } 2007 \text { [18] } \\
\text { Denmark 2005-2006 }\end{array}$} & \multirow[t]{2}{*}{$\begin{array}{l}\text { TKR, OA with planned spinal } \\
\text { anaesthesia } 77 \text { ( } 40: 37) \\
70: 72 \text { years } 63: 60 \% \text { female }\end{array}$} & $\begin{array}{l}\text { Spinal and after surgery immediate release } \\
\text { oxycodone and intravenous morphine if } \\
\text { required }\end{array}$ & \\
\hline & & $\begin{array}{l}152 \mathrm{ml} \text { solution containing } 300 \mathrm{mg} \text { ropivacaine, } \\
30 \mathrm{mg} \text { ketorolac and } 0.5 \mathrm{mg} \text { epinephrine } \\
\text { infiltrated during surgery. Further infiltrate through } \\
\text { catheter intra-articularly on day of surgery and } \\
\text { post-operative day } 1 .\end{array}$ & $\begin{array}{l}\text { Femoral nerve block prior to } \\
\text { spinal anaesthesia No placebo } \\
\text { infiltration Post-surgical } \\
\text { continuous femoral nerve } \\
\text { block }\end{array}$ \\
\hline \multirow{2}{*}{$\begin{array}{l}\text { Vendittoli et al. } 2006 \text { [39] } \\
\text { Canada 2003-2004 }\end{array}$} & \multirow{2}{*}{$\begin{array}{l}\text { TKR, } 95.2 \% \text { OA N }=42(22: 20) \\
\text { Ages not specified } 73: 70 \% \\
\text { female }\end{array}$} & Spinal anaesthesia, PCA morphine & \\
\hline & & $\begin{array}{l}160 \mathrm{ml} \text { solution containing in total } 400 \mathrm{mg} \\
\text { ropivacaine, } 30 \mathrm{mg} \text { ketorolac and } 0.5 \mathrm{ml} \\
\text { adrenaline (1:1000) infiltrated during surgery. } \\
\text { Infiltrate through catheter intra-articularly on day1. }\end{array}$ & No placebo infiltration \\
\hline \multirow{2}{*}{$\begin{array}{l}\text { Zhang et al. } 2007 \text { [41] China } \\
\text { 2006-2007 }\end{array}$} & \multirow{2}{*}{$\begin{array}{l}\text { TKR, unilateral } N=60(30: 30) \\
\text { Overall } 68 \text { years } 83: 80 \% \text { female }\end{array}$} & PCA morphine & \\
\hline & & $\begin{array}{l}60 \mathrm{ml} \text { solution containing } 0.25 \% \text { bupivacaine, } \\
\text { epinephrine (1:200,000) and } 10 \mathrm{mg} \text { morphine } \\
\text { infiltrated during surgery }\end{array}$ & No placebo injection \\
\hline
\end{tabular}

72 hours and to discharge VAS at rest and during knee flexion, morphine consumption, functional recovery, length of stay, satisfaction, mobilisation including walking distance, adverse events $2(1: 1)$

lost to follow up Low risk of bias

4 days and to discharge VAS pain, morphine consumption, satisfaction, mobilisation getting out of bed without assistance, walking with crutches), functional recovery, length of hospital stay 21 (13:8) patients lost to follow up data reported Possible bias (large uneven losses to follow up, group allocation by date of birth)

4 days and to discharge NRS pain, opioid consumption, mobilisation (able to walk $>3$ metres, able to hold quadriceps tension for $>5 \mathrm{sec}$ ), length of hospital stay, adverse events and complications 4 (3:1) patients lost to follow up Unclear (blinding of outcome assessment)

5 days and to discharge VAS pain at rest and during physiotherapy exercise, PCA morphine consumption, functional recovery, side effects No losses to follow up described Low risk of bias

72 hours VAS pain at rest and activity, functional recovery No losses to follow up described Unclear (blinding of outcome assessment)

TKR: total knee replacement, THR: total hip replacement, OA: osteoarthritis, RA: rheumatoid arthritis, PCA: patient controlled analgesia, FNB: Femoral Nerve Block, VAS: visual analogue scale, NRS: numerical response scale, ROM: range of motion, WOMAC: Western Ontario and McMaster Universities Arthritis Index. 
Table 3 Meta-analyses of pain and length of hospital stay by anaesthetic regimen compared with controls using a random effects model

\begin{tabular}{|c|c|c|c|c|c|c|c|}
\hline TOTAL HIP REPLACEMENT STUDIES & $\mathbf{N}$ & Measure & Pooled effect size & Confidence Interval & P-value & $I^{2}(\%)$ & $\tau^{2}$ \\
\hline \multicolumn{8}{|c|}{$(A+B)$ Any wound infiltration analgesia + usual anaesthesia vs Usual anaesthesia } \\
\hline Pain at rest at $24 \mathrm{~h}$ & 12 & SMD & -0.605 & $(-1.051,-0.160)$ & 0.0078 & 89 & 0.541 \\
\hline Pain during activity at $24 \mathrm{~h}$ & 9 & SMD & -0.848 & $(-1.450,-0.246)$ & 0.0058 & 92 & 0.765 \\
\hline Pain at rest at $48 \mathrm{~h}$ & 11 & SMD & -0.285 & $(-0.520,-0.050)$ & 0.018 & 58 & 0.09 \\
\hline Pain during activity at $48 \mathrm{~h}$ & 8 & SMD & -0.432 & $(-0.776,-0.089)$ & 0.014 & 71 & 0.171 \\
\hline Length of hospital stay & 9 & MD & -0.829 & $(-1.540,-0.118)$ & 0.022 & 84 & 0.866 \\
\hline \multicolumn{8}{|c|}{ (A) Wound infiltration analgesia + usual analgesia vs Usual anaesthesia } \\
\hline Pain at rest $24 \mathrm{~h}$ post-op & 7 & SMD & -0.633 & $(-1.208,-0.059)$ & 0.031 & 90 & 0.529 \\
\hline Pain during activity $24 \mathrm{~h}$ post-op & 4 & SMD & -0.241 & $(-0.637,0.155)$ & 0.23 & 68 & 0.11 \\
\hline Pain at rest $48 \mathrm{~h}$ post-op & 6 & SMD & -0.134 & $(-0.348,0.080)$ & 0.22 & 19 & 0.014 \\
\hline Pain during activity 48 h post-op & 3 & SMD & -0.225 & $(-0.559,0.109)$ & 0.19 & 35 & 0.03 \\
\hline Length of Hospital Stay & 5 & MD & -0.257 & $(-0.622,0.108)$ & 0.17 & 14 & 0.029 \\
\hline
\end{tabular}

(B) Wound infiltration analgesia + post closure analgesia + usual anaesthesia vs Usual anaesthesia

\begin{tabular}{|c|c|c|c|c|c|c|c|}
\hline Pain at rest $24 \mathrm{~h}$ post-op & 5 & SMD & -0.572 & $(-1.383,0.240)$ & 0.17 & 90 & 0.767 \\
\hline Pain during activity $24 \mathrm{~h}$ post-op & 5 & SMD & -1.378 & $(-2.499,-0.257)$ & 0.016 & 94 & 1.525 \\
\hline Pain at rest $48 \mathrm{~h}$ post-op & 5 & SMD & -0.489 & $(-0.963,-0.015)$ & 0.043 & 73 & 0.209 \\
\hline Pain during activity $48 \mathrm{~h}$ post-op & 5 & SMD & -0.599 & $(-1.158,-0.040)$ & 0.036 & 80 & 0.319 \\
\hline Length of Hospital Stay & 4 & $\mathrm{MD}$ & -1.117 & $(-2.474,0.239)$ & 0.11 & 88 & 1.621 \\
\hline TOTAL KNEE REPLACEMENT STUDIES & $\mathbf{N}$ & Measure & Pooled effect size & Confidence Interval & P-value & $12(\%)$ & $\tau 2$ \\
\hline \multicolumn{8}{|c|}{$(A+B)$ Any wound infiltration analgesia + usual anaesthesia vs Usual anaesthesia } \\
\hline Pain at rest at $24 \mathrm{~h}$ & 12 & SMD & -0.398 & $(-0.576,-0.219)$ & $p<0.001$ & 32 & 0.032 \\
\hline Pain during activity at $24 \mathrm{~h}$ & 12 & SMD & -0.453 & $(-0.671,-0.235)$ & $p<0.001$ & 54 & 0.078 \\
\hline Pain at rest at $48 \mathrm{~h}$ & 12 & SMD & -0.325 & $(-0.546,-0.103)$ & 0.0041 & 56 & 0.084 \\
\hline Pain during activity at $48 \mathrm{~h}$ & 11 & SMD & -0.273 & $(-0.500,-0.046)$ & 0.018 & 56 & 0.081 \\
\hline Length of hospital stay & 8 & MD & -0.866 & $(-1.622,-0.109)$ & 0.025 & 77 & 0.805 \\
\hline \multicolumn{8}{|c|}{ (A) Wound infiltration analgesia with no additional post closure analgesia + usual anaesthesia vs Usual anaesthesia } \\
\hline Pain at rest 24 h post-op & 6 & SMD & -0.248 & $(-0.452,-0.044)$ & 0.017 & 14 & 0.009 \\
\hline Pain during activity $24 \mathrm{~h}$ post-op & 6 & SMD & -0.283 & $(-0.470,-0.096)$ & 0.0031 & 0 & 0 \\
\hline Pain at rest $48 \mathrm{~h}$ post-op & 6 & SMD & -0.155 & $(-0.458,0.148)$ & 0.32 & 61 & 0.086 \\
\hline Pain during activity $48 \mathrm{~h}$ post-op & 6 & SMD & -0.077 & $(-0.263,0.110)$ & 0.42 & 0 & 0 \\
\hline Length of Hospital Stay & 1 & $\mathrm{MD}$ & 0.092 & $(-0.890,1.073)$ & 0.85 & 100 & $p<0.00$ \\
\hline
\end{tabular}

(B) Wound infiltration analgesia + post wound closure analgesia + usual anaesthesia vs Usual anaesthesia

$\begin{array}{lllllccc}\text { Pain at rest 24 h post-op } & 6 & \text { SMD } & -0.587 & (-0.829,-0.346) & p<0.001 & 9 & 0.008 \\ \text { Pain during activity 24 h post-op } & 6 & \text { SMD } & -0.693 & (-1.152,-0.234) & 0.0031 & 74 & 0.24 \\ \text { Pain at rest 48 h post-op } & 6 & \text { SMD } & -0.52 & (-0.778,-0.262) & p<0.001 & 21 & 0.022 \\ \text { Pain during activity 48 h post-op } & 5 & \text { SMD } & -0.594 & (-0.997,-0.191) & 0.0039 & 61 & 0.128 \\ \text { Length of Hospital Stay } & 7 & \text { MD } & -1.023 & (-1.822,-0.224) & 0.012 & 76 & 0.761\end{array}$

(C) Wound infiltration analgesia + post wound closure analgesia + usual anaesthesia vs Femoral nerve block + usual anaesthesia

$\begin{array}{lllllccc}\text { Pain at rest 24 h post-op } & 3 & \text { SMD } & 0.253 & (-0.514,1.021) & 0.52 & 81 & 0.37 \\ \text { Pain during activity 24 h post-op } & 3 & \text { SMD } & 0 & (-0.317,0.317) & 1 & 0 \\ \text { Pain at rest 48 h post-op } & 2 & \text { SMD } & 0.254 & (-0.429,0.937) & 0.47 & 67 & 0.166 \\ \text { Pain during activity 48 h post-op } & 2 & \text { SMD } & -0.073 & (-0.446,0.299) & 0.7 & 0 & 0 \\ \text { Length of Hospital Stay } & 2 & \text { MD } & 0.07 & (-0.838,0.978) & 0.88 & 0\end{array}$


Table 3 Meta-analyses of pain and length of hospital stay by anaesthetic regimen compared with controls using a random effects model (Continued)

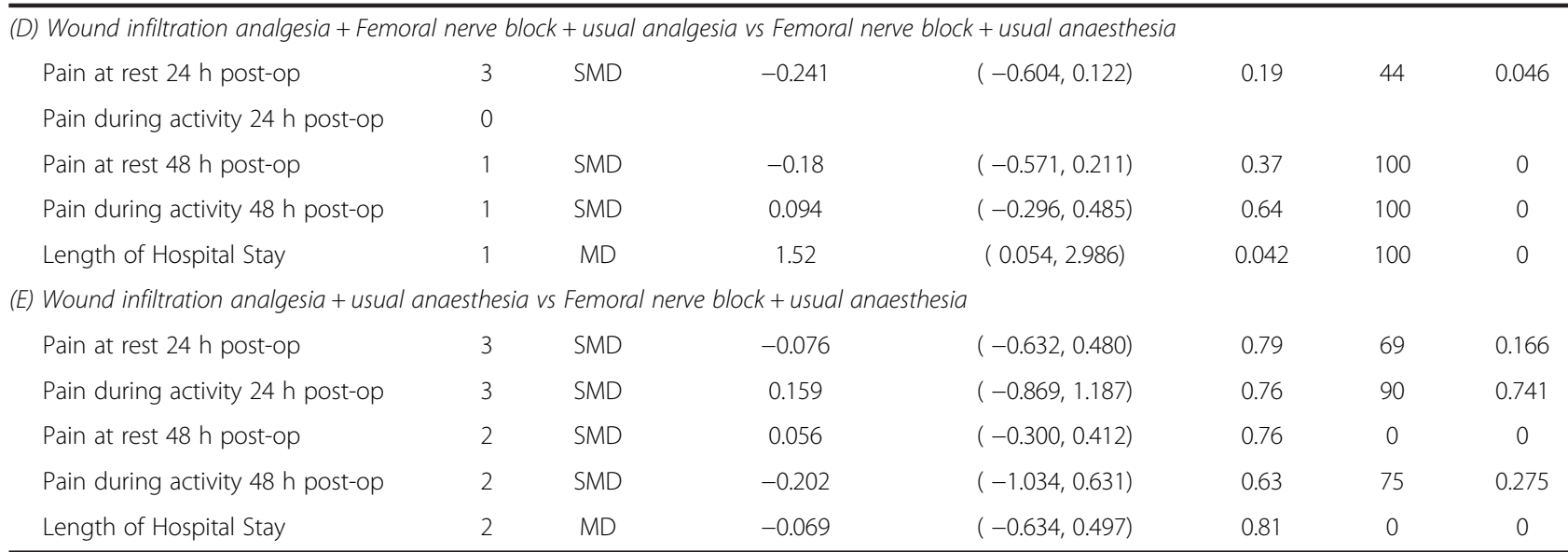

$\mathrm{N}$ : Number of studies in meta-analysis. Two studies (Essving 2011, Parvataneni 2007 hip and knee) reported mean pain scores for the first 48 hours. These data were duplicated for the 24 and 48 hour outcomes. One study (Andersen LJ, 2007) reported WOMAC pain scores, a composite measure of pain taken at rest and during activity. These data were duplicated for rest and during activity outcomes.

infiltration group. In a study where control patients received additional intrathecal morphine, there was no difference in pain outcomes at any time point [37].

Heterogeneity measured by the $\mathrm{I}^{2}$ and $\tau^{2}$ statistics was high, and separating the analysis for $\mathrm{A}$ and $\mathrm{B}$ groups did not appear to reduce this heterogeneity. Restricting the analysis to studies with low risk of bias gave a marginally smaller estimate of reduction in pain at 24 hours at rest by an average of SMD -0.49 (95\% CI $-0.89,-0.09$; $\mathrm{p}=0.017$ ), but during activity average pain reduction

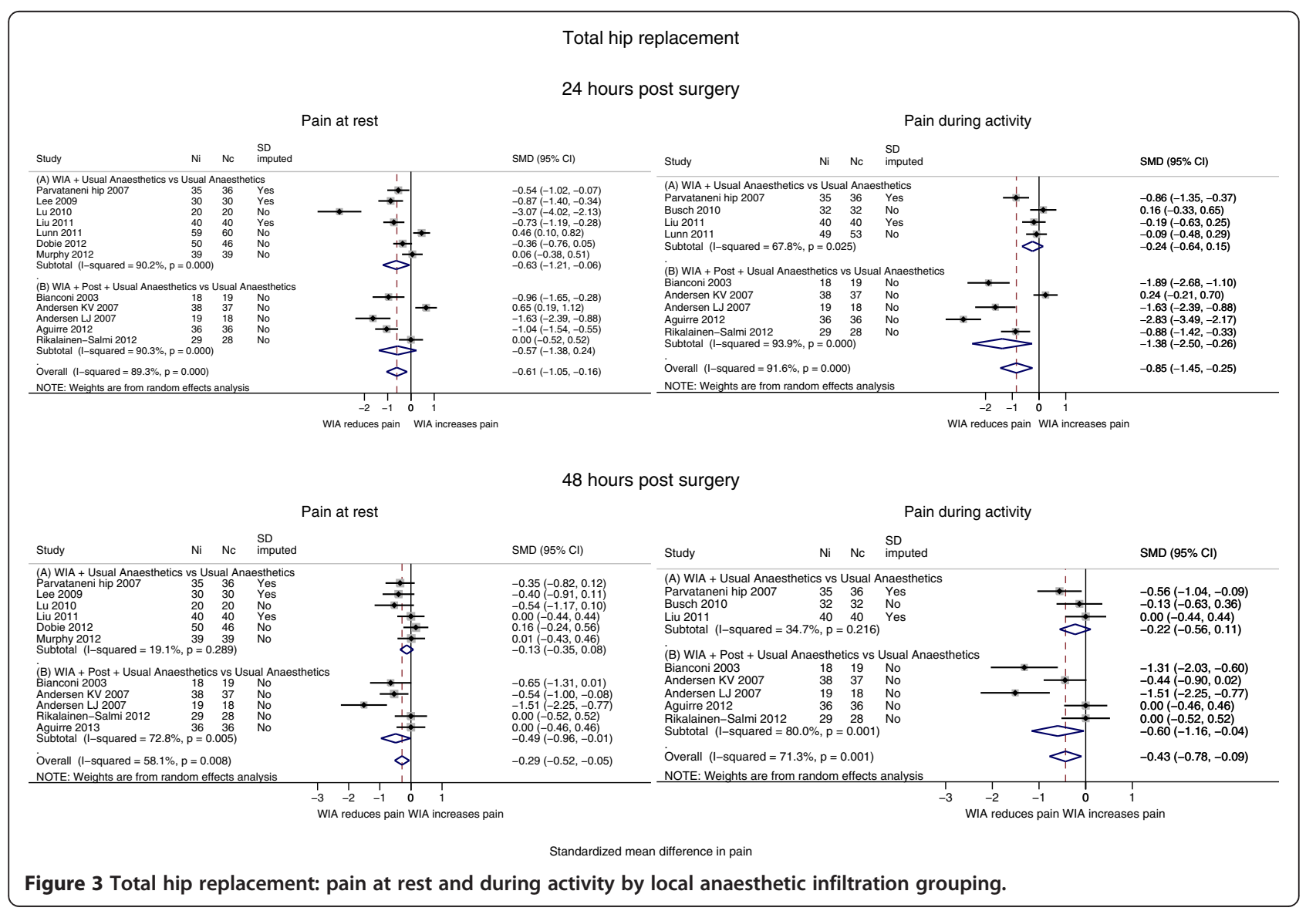


appeared greater at SMD -0.99 (95\% CI -1.64, -0.35 ; $\mathrm{p}=0.003$ ), corresponding to 28 points on a 100 point scale.

\section{Opioid consumption}

In all 11 studies reporting an outcome, opioid consumption was reduced in patients receiving local anaesthetic infiltration compared with controls [10,16,27,30-37]. This difference ranged from 12 to $92 \%$. There was no suggestion of different effects in groups with or without additional analgesia through a catheter or injection.

In the studies where control patients received epidural or intrathecal analgesia, patients receiving local anaesthetic infiltration consumed $20 \%$ and $12 \%$ less morphine, respectively.

\section{Mobilisation}

Several different measures of mobilisation were reported. In three studies patients receiving local anaesthetic infiltration with no additional post-operative component achieved a straight leg raise earlier than control patients $[28,29,32]$. More patients were able to walk during the first post-operative day in two studies where additional post-operative analgesia was provided through a catheter $[16,37]$. In one study with no additional analgesia, with the exception of those with adverse events, all patients were mobilised on the first post-operative day [35]. However, in patients receiving local anaesthetic infiltration, walking speed over six metres at a two-day functional assessment was improved.

In one study, $35 \%$ of patients receiving local anaesthetic infiltration were able to walk after 8 hours compared with $87 \%$ of control patients receiving an epidural infusion. In the study where control patients received intrathecal morphine, 33\% of these patients could walk further than 5 metres on the first post-operative day compared with $71 \%$ of patients receiving local anaesthetic infiltration.

\section{Length of hospital stay}

As shown in Table 3 and Figure 4, patients receiving local anaesthetic infiltration spent an average 0.83 fewer days $(95 \%$ CI $0.12,1.54$ days; $\mathrm{p}=0.022$ ) in hospital compared with controls. Benefit was largely driven by interventions with additional analgesia through a catheter or injection (B comparisons). Heterogeneity across studies was high $\left(\mathrm{I}^{2}=84 \%\right)$, mainly in studies with additional post-operative analgesia.

When the comparison group received an epidural infusion [16], patients with local anaesthetic infiltration had on average a two day shorter hospital stay. In the

\section{Hospital length of stay}

Total hip replacement

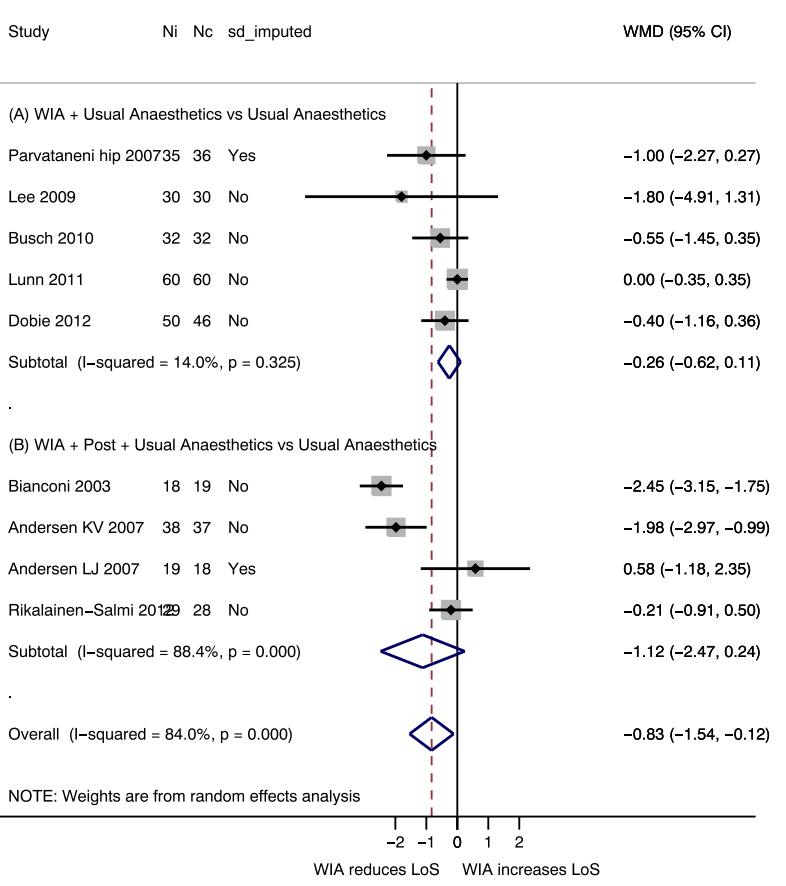

Total knee replacement

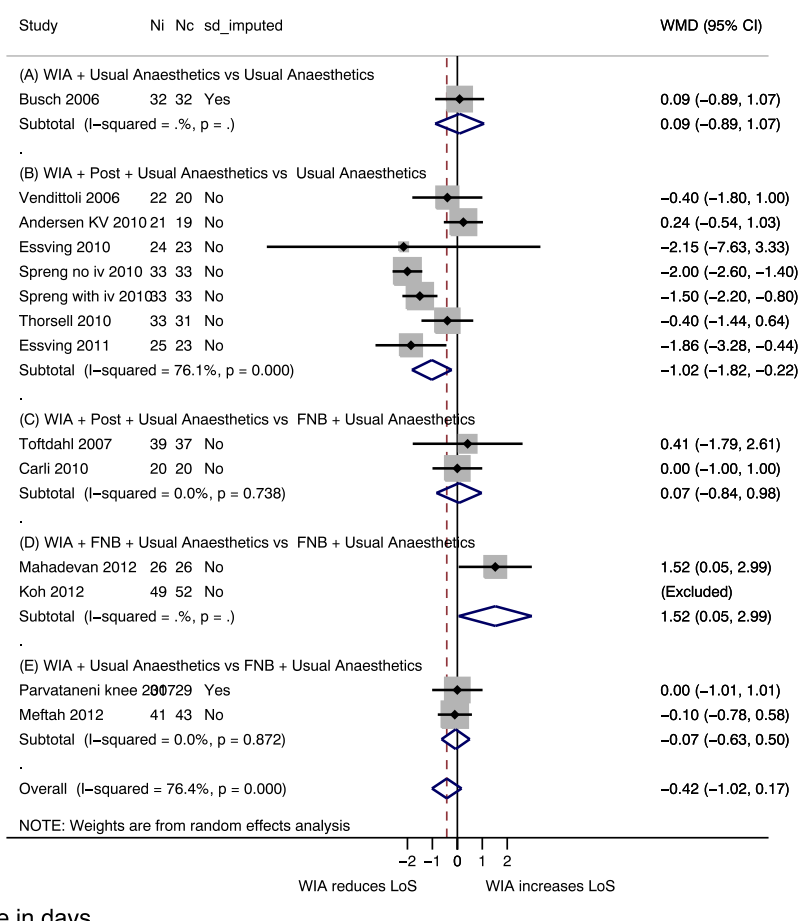

Figure 4 Length of hospital stay by local anaesthetic infiltration grouping. 
study where the comparison group received intrathecal morphine [37], there was no clear difference in discharge times.

\section{Complications}

The Peto OR for a major complication in patients with local anaesthetic infiltration compared with controls was 0.30 (95\% CI 0.05, 1.77; p =0.18), but this is weak evidence, based on five major complications in 896 patients. Five deep infections were reported, four in local anaesthetic infiltration patients and one in controls, Peto OR 3.47 (95\% CI 0.58, 20.81; $\mathrm{p}=0.17$ ). Four infections occurred in the 218 patients who received post-closure delivery of infiltrate through a catheter.

The incidence of vomiting was reduced in patients receiving local anaesthetic infiltration in five studies with 309 patients with data, Peto OR 0.46 (95\% CI 0.27, 0.80; $\mathrm{p}=0.006)$.

\section{Long-term outcomes}

Five studies reported long-term outcomes. Andersen and colleagues reported a trend for improved Western Ontario and McMaster Universities Osteoarthritis Index (WOMAC) pain scores at six weeks in local anaesthetic infiltration patients compared with controls [27]. At eight week follow up, Rikalainen-Salmi and colleagues reported no differences in mobilisation, intensity or duration of pain [37]. Parvataneni and colleagues reported that VAS pain scores were "comparable between groups" at 3 months [28]. Similarly, Aguirre and colleagues reported no difference in analgesic consumption or pain during normal daily activities between groups at 3 months [34]. Busch and colleagues reported a trend for improved WOMAC score at two years in local anaesthetic infiltration patients compared with controls [30].

\section{Total knee replacement}

Overall there were 23 studies including 1,439 patients with TKR [18,28,38-56]. Study characteristics and our overall risk of bias assessment are summarised in Table 2 . The mean number of patients randomised was 63 (range 32-101). We assessed that 17 studies were at low risk of bias and that five studies had unclear risk of bias based on uncertainty about blinding of outcome assessments. One study was assessed to be at high risk of bias due to a large uneven loss to follow up between randomised groups.

\section{Pain}

As shown in Table 3 and Figure 5, there was strong evidence that on average across 12 studies [39-42,44,46-48,51,52], patients receiving local anaesthetic infiltration ( $\mathrm{A}+\mathrm{B}$ studies) reported lower pain at rest compared with controls at 24 and 48 hours. For example, pain at rest at 24 hours and during activity at 48 hours was reduced by SMD -0.40 $(95 \% \mathrm{CI}-0.58,-0.22 ; \mathrm{p}<0.001)$ and SMD $-0.27(95 \%$ CI $-0.50,-0.05 ; \mathrm{p}=0.018)$, respectively. This reflected reductions in pain at rest at 24 hours by an average of 10 points $(95 \%$ CI 6,$15 ; \mathrm{p}<0.001)$ and during activity at 48 hours by 8 points $(95 \%$ CI $1.5,15 ; \mathrm{p}=0.018)$ on a 100 point scale.

Heterogeneity was moderate to low. When we restricted analyses to studies assessed as low risk of bias, pain outcome estimates were slightly attenuated towards zero.

We additionally performed separate analyses according to whether additional analgesia was delivered after wound closure through a catheter or injection. In the six studies with no further analgesia (A studies) [40-42,47,52], pain at 24 hours was lower at rest by SMD -0.25 (95\% CI $-0.45,-0.04 ; \mathrm{p}=0.017)$, and during activity by SMD -0.28 (95\% CI $-0.47,-0.10 ; \mathrm{p}=0.003)$. At 48 hours, pooled effect estimates favoured local anaesthetic infiltration but there was no strong evidence that the intervention was beneficial.

In six studies with additional analgesia delivered after wound closure (B studies) $[39,44,46,48,51]$, pain was reduced on average at 24 hours at rest by SMD -0.59 $(95 \%$ CI $-0.83,-0.35 ; \mathrm{p}<0.001)$ and during activity by SMD $-0.69(95 \%$ CI $-1.15,-0.23 ; \mathrm{p}=0.003)$. At 48 hours, pain was reduced at rest by SMD -0.52 $(95 \% \mathrm{CI}-0.78,-0.26 ; \mathrm{p}<0.001)$ and during activity by SMD -0.59 (95\% CI $-1.00,-0.19 ; \mathrm{p}=0.004)$.

In six studies comparing local anaesthetic infiltration with or without additional post-closure analgesia against femoral nerve block, there was no evidence for improvement in pain at any time point $[18,28,45,50,55,56]$. In three studies where both randomised groups received a femoral nerve block (D studies) [43,53,54], there was no evidence for added benefit of local anaesthetic infiltration for pain outcomes.

In eight comparisons between local anaesthetic infiltration with controls $[38,39,44,46,48,49,51]$, additional ketoralac was included in the wound infiltrate. In seven comparisons with data $[38,39,44,46,48,51]$, there was strong evidence that patients receiving additional analgesia in the infiltrate on average had lower pain compared with controls. For example, pain was reduced on average at rest at 24 hours by SMD -0.68 (95\% CI $-0.94,-0.42 ; \mathrm{p}<0.001)$ and during activity at 48 hours by SMD $-0.59(95 \% \mathrm{CI}-1.01,-0.17 ; \mathrm{p}=0.006)$, equivalent to a reduction of 17 and 30 points respectively on a 100 point scale.

In four studies, control patients received either an epidural infusion $[44,48,49]$ or intrathecal morphine [51]. Results of all studies supported a reduction in pain for patients receiving local anaesthetic infiltration compared with epidural or intrathecal morphine. 


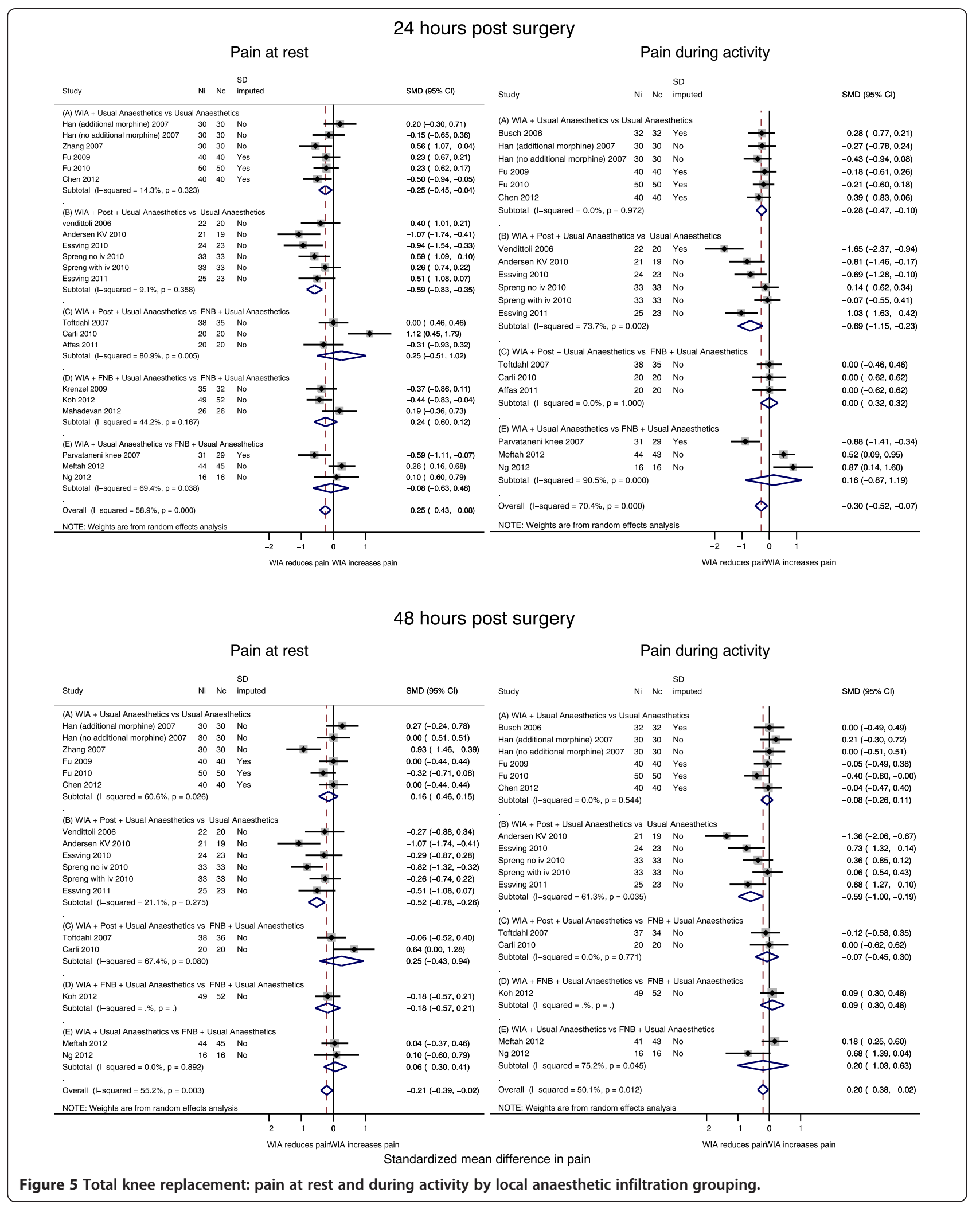

\section{Opioid consumption}

In all four studies reporting opioid consumption, this was reduced by $35-40 \%$ in patients receiving wound infiltration with no additional post-closure analgesia [38,42,47,52], and by $32-52 \%$ in three studies with additional post-closure analgesia, compared with controls [39,46,51]. 
In six studies where the control group or both groups received femoral nerve block, there was little difference in opioid consumption between randomised groups $[18,28,43,45,50,53]$.

In four studies where patients receiving wound infiltration with further post-closure analgesia were compared with patients receiving epidural anaesthesia, there was no consistent difference between groups $[44,48,49]$.

\section{Mobilisation}

Nineteen studies reported a mobilisation outcome. In four studies, patients receiving local anaesthetic infiltration had reduced time to achieve a straight leg raise by an estimated $44-50 \%[42,47,52]$ or were more likely to achieve a straight leg raise on the first post-operative day compared with control patients [28]. In two studies with femoral nerve block given to all patients, more patients receiving local anaesthetic infiltration were able to achieve a straight leg raise during the first post-operative day $[43,53]$.

In four out of five studies, patients receiving local anaesthetic infiltration achieved better knee flexion [39,40,47,54]. In four studies $[44,46,51,55]$, ambulation was part of discharge readiness criteria. These criteria were met earlier in local anaesthetic infiltration patients in three studies $[44,46,51]$, but were similar in one study where control patients received a femoral nerve block [55].

Improvements to diverse walking goals were reported in patients receiving local anaesthetic infiltration in three studies where some or all of the comparison group patients received epidural analgesia $[48,49]$. When the comparison group or all patients received femoral nerve block, walking goals were achieved earlier after local anaesthetic infiltration in one study [18], with trends for benefit in two studies $[43,45]$.

\section{Length of hospital stay}

Data on length of hospital stay were available for 8 studies comparing local anaesthetic infiltration with controls $[38,39,44,46,48,49,51]$, of which seven had a post-closure analgesia component. As shown in Table 3 and Figure 4, length of hospital stay was reduced in patients receiving local anaesthetic infiltration and additional post-closure delivery (B studies) by 1.0 day on average (95\% CI 0.2, 1.8 days; $\mathrm{p}=0.012$ ) compared with controls. In the one (A) study with no post-closure analgesia component there was no difference in length of hospital stay.

In three studies where the comparison group received femoral nerve block $[18,45,55]$, there was no suggestion of a difference in length of stay. In one study in which all randomised patients received a femoral nerve block, the length of hospital stay was about 1.5 days shorter in the control patients who also received a sciatic nerve block [54].
In four studies where the control group received epidural analgesia $[44,48,49]$, length of hospital stay was reduced in patients receiving local anaesthetic infiltration with the exception of one study in which the authors reported shorter time to fulfilment of discharge criteria [44].

\section{Complications}

Based on 11 events, the Peto OR for a major complication was $1.17(95 \%$ CI $0.35,3.86 ; \mathrm{p}=0.80)$ in patients receiving local anaesthetic infiltration compared with controls. There were two deep infections in intervention patients [18,44], and one in control groups [48], Peto OR 1.85 (95\% CI 0.19, $17.83 ; \mathrm{p}=0.59$ ). Two infections occurred in the $287 \mathrm{pa}-$ tients who received post-closure delivery of infiltrate through a catheter.

Excluding one intervention with additional morphine [40], there was evidence that the incidence of vomiting was lower in local anaesthetic infiltration patients compared with controls in eight studies with 548 patients [40,42,46-48,51,52], Peto OR 0.56 (95\% CI 0.39, 0.80; $\mathrm{p}=0.002)$.

\section{Long-term outcomes}

Five studies reported outcomes measured at six weeks $[38,45]$, or three months $[28,46,51]$. Busch and colleagues showed a trend for improved pain at 6 weeks favouring the intervention group [38]. Parvataneni and colleagues reported comparable pain scores between groups at 3 months [28]. In the studies of Essving and colleagues, there were no differences between median Oxford Knee Scores at 3 months $[46,51]$.

Carli and colleagues reported poorer WOMAC scores after 6 weeks in patients receiving local anaesthetic infiltration compared with the control group who received femoral nerve block [45].

\section{Discussion}

Our systematic review and meta-analyses represent a comprehensive overview of evaluations of the effectiveness of peri-operative local anaesthetic infiltration in THR and TKR. Systematic reviews allow for a more objective appraisal than traditional narrative reviews [57], which are often biased in their selection of studies and thus may be unreliable in their recommendations of interventions [58]. Extensive efforts to acquire information from authors on unpublished outcomes and variance data allowed us to apply methods for meta-analyses of continuous and skewed outcomes and to produce more robust results for some outcomes than could be achieved with a purely narrative synthesis.

In conducting this systematic review we recognised the problems that can arise when small studies are included in meta-analyses [59]. In this review it is noteworthy that 28 out of 35 studies (80\%) reported a power 
calculation. Review of studies with data largely collected in highly controlled conditions in the peri-operative and early post-operative period benefitted from low losses to follow up and more complete data. With the exception of one study where the authors acknowledged uneven losses to follow up due to inadequate protocols, the main risk of bias arose from uncertainty about blind outcome assessment. As most studies reported VAS pain and other self-reported outcomes, we believe that the evidence base on short-term outcomes is of reasonably good quality.

Pain after THR was reduced for patients receiving local anaesthetic infiltration, with patients experiencing less pain at rest at 24 hours and during activity at 48 hours equivalent to about 12 and 10 points on a 100 point pain intensity scale. In musculoskeletal settings, VAS pain changes of 11 [60], and 14 [61], are considered clinically significant [62]. Patients receiving local anaesthetic infiltration had lower pain levels after their THR, used less opioid medication and had a reduced incidence of vomiting and nausea. This may explain the early mobilisation and earlier discharge of patients who received local anaesthetic infiltration, irrespective of alternative pain management strategies. Opioid medication is a key strategy in the management of post-surgical pain but its use can delay mobilisation and rehabilitation [63].

Pain after TKR was also reduced for patients receiving local anaesthetic infiltration compared with controls, with less pain at rest at 24 hours and after 48 hours during activity, equivalent to reductions of about 10 and 8 points on a 100 point pain intensity scale. Opioid consumption was reduced compared with untreated control patients and there was a general observation of early mobilisation, reduced vomiting and nausea, and early hospital discharge. Inclusion of the non-steroidal antiinflammatory agent ketoralac in the infiltrate seemed to enhance post-operative pain relief.

When compared with alternative regimens, results were not so clear. Pain levels after TKR were broadly similar when femoral nerve block was included in the general analgesia regimen or as a comparator. Likewise, opioid consumption was similar. There was some suggestion of benefit for earlier mobilisation, but length of hospital stay was not reduced in patients receiving local anaesthetic infiltration. Femoral nerve block is a well established method of providing analgesia after TKR and is associated with reduced opioid requirement and thus fewer side effects such as nausea and vomiting. However, femoral nerve block is associated with decreased quadriceps function for a time and an increased risk of falls $[64,65]$.

In studies in patients receiving TKR where control groups received epidural or intrathecal analgesia, benefit was observed for reduced pain in patients receiving local anaesthetic infiltration. Opioid consumption did not differ between groups but mobilisation and hospital discharge were achieved earlier in patients receiving local anaesthetic infiltration.

The improvement in pain control and shorter hospital stay was greatest for patients receiving additional analgesia through a catheter or by injection. However, we observed a small but potentially important increase in rates of serious infection, particularly in patients receiving further infiltrate through a catheter post-wound closure. Across THR and TKR studies, there were eight cases of deep infection requiring surgical debridement or revision. Six of these were in patients randomised to wound infiltration analgesia with additional analgesia through a post-surgical catheter. Indeed, all patients with deep infection had been randomised to receiving a catheter although researchers reported that catheters in control groups were not inserted into the joint capsule. The overall rate of infection in patients with THR or TKR randomised into wound infiltration analgesia studies was $0.34 \%$ and in patients receiving an active catheter the rate was $1.4 \%$.

Few studies in patients with THR or TKR reported long-term follow up of patients and results were equivocal. Acute post-operative pain is an important risk factor for long-term pain [66,67], and deserves appropriate consideration in future studies of peri-operative pain control.

Our study has limitations. Although meta-analyses performed were enhanced by extensive contact with authors, imputation was required for some measures of variability. The skewed nature of hospital stay required transformation under assumptions of a lognormal distribution [26]. For opioid consumption and mobilization there was insufficient consistency in measures reported to conduct anything but a systematic narrative overview. We noted a range of analgesia regimens, with different studies making different comparisons, particularly for TKR. We considered it unnecessary to make indirect comparisons between regimens, since direct evidence was available for all the comparisons of interest.

A further limitation of meta-analyses in a highly active field of research such as wound infiltration analgesia is that they may become out of date quickly. Their value is emphasised, however, in a widely cited example when studies of streptokinase in acute coronary heart disease were conducted long after a critical mass of evidence had been obtained from meta-analysis showing benefit for patients [68]. Prior to submission, we updated searches in December 2013 and identified 12 new studies, three in patients with THR and nine in patients with TKR. Our results for local anaesthetic infiltration in patients receiving THR were supported with reduced pain compared with untreated control [69-71], or similar pain 
compared with epidural analgesia [71]. The results of our meta-analyses in patients receiving TKR were also supported with improved early pain control in patients receiving local anaesthetic infiltration [72]; further pain reduction with added ketoralac $[73,74]$ but not steroid [75]; and uncertainty when compared with femoral nerve block [76-80].

Our results show that local anaesthetic infiltration is effective in reducing short-term pain after THR and TKR when compared with no anaesthetic infiltration. The effect of local anaesthetic infiltration is enhanced with the addition of post-closure analgesia, although this needs to be considered in light of the infection risks associated with catheters [81]. In TKR, there may be no added benefit to femoral nerve block. Further studies are in progress to assess long-term effectiveness of local anaesthetic infiltration [11].

\section{Conclusions}

Our systematic review and meta-analysis shows that inclusion of local anaesthetic infiltration in a multimodal anaesthesia regimen is effective in reducing shortterm pain and hospital stay in patients receiving THR and TKR. Enhanced pain control was observed when additional analgesia was provided after wound closure through a catheter but benefit should be weighed against a possible infection risk. For patients with TKR, inclusion of the non-steroidal anti-inflammatory agent ketoralac in the infiltrate seemed to enhance pain relief. There was no evidence of pain control additional to that provided by femoral nerve block in patients receiving TKR. Few studies reported long-term outcomes and future research should assess whether local anaesthetic infiltration can affect the development of long-term post-surgical pain.

\section{Additional files}

Additional file 1: PRISMA checklist.

Additional file 2: Cochrane risk of bias table $(\checkmark$ low risk, $X$ risk; $\sim$ no reason to assume bias).

\section{Abbreviations}

THR: Total hip replacement; TKR: Total knee replacement; RCT: Randomised controlled trial; SMD: Standardised mean difference; MD: Mean difference; VAS: Visual analogue scale; OR: Odds ratio; WOMAC: Western Ontario and McMaster Universities Osteoarthritis Index.

\section{Competing interests}

The authors declare that they have no competing of interests.

\section{Authors' contributions}

$A D B, E M, A W B$ and MP designed the study and produced the search strategy. EM and ADB performed the systematic review as second and first reviewers respectively, conducted the searches, screened abstracts and titles, assessed inclusion and exclusion criteria, produced data collection forms and extracted data, assessed study quality and contacted authors. AWB and MP advised on inclusion/ exclusion criteria and subgroup analyses. HEJ provided statistical guidance. EM and HEJ performed meta-analyses of continuous and skewed outcomes (pain and length of stay). KTE and ADB collected complications data and performed meta-analyses of complications. EM, $\mathrm{ADB}$ and HEJ drafted the article with critical revisions from AWB, MP and KTE. All authors read and approved the final manuscript.

\section{Acknowledgements}

We contacted study authors and are grateful for the additional information provided by: Constant Busch, Damien Bennett, Damien Byrne, Gary Minto, In Jun Koh, Karen Toftdahl Bjørnholdt, Martin Thorsell, Michael Whitehouse, Per Essving and Per Wretenberg. We further acknowledge Peiliang Fu and Yi ChenYun-Li Zhu for clarifying previously reported data. We would like to thank Joanna Thorn for helpful edits.

\section{Funding}

Role of the funding source

The sponsor of the study had no role in study design, data collection, data analysis, data interpretation, or writing of the report. All authors had full access to all the data in the study and had final responsibility for the decision to submit for publication.

This article outlines independent research commissioned by the National Institute for Health Research (NIHR) in England under its Programme Grants for Applied Research funding scheme (RP-PG-0407-10070). The views expressed are those of the authors and not necessarily those of the NHS, the NIHR or the Department of Health.

\section{Author details}

${ }^{1}$ School of Social and Community Medicine, University of Bristol, Canynge Hall, 39 Whatley Road, Bristol BS8 2PS, UK. ${ }^{2}$ Musculoskeletal Research Unit, School of Clinical Sciences, University of Bristol, Avon Orthopaedic Centre, Southmead Hospital, Bristol BS10 5NB, UK. ${ }^{3}$ North Bristol Healthcare Trust, Southmead Hospital, Westbury-on-Trym, Bristol BS10 5NB, UK.

Received: 21 March 2014 Accepted: 30 June 2014

Published: 5 July 2014

References

1. National Joint Registry for England and Wales: 10th Annual Report. Hemel Hempstead: NJR; 2013

2. Centers for Disease Control and Prevention: Number of all-listed procedures for discharges from short-stay hospitals, by procedure category and age: United States. In CDCANCHS National Hospital Discharge Survey. Atlanta, GA: Centers for Disease Control and Prevention; 2010.

3. Wylde V, Rooker J, Halliday L, Blom A: Acute postoperative pain at rest after hip and knee arthroplasty: severity, sensory qualities and impact on sleep. Orthop Traumatol Surg Res 2011, 97:139-144.

4. Beswick AD, Wylde V, Gooberman-Hill R, Blom A, Dieppe P: What proportion of patients report long-term pain after total hip or knee replacement for osteoarthritis? A systematic review of prospective studies in unselected patients. BMJ Open 2012, 2:e000435.

5. Chan EY, Blyth FM, Nairn L, Fransen M: Acute postoperative pain following hospital discharge after total knee arthroplasty. Osteoarthritis Cartilage 2013, 21:1257-1263

6. Jin F, Chung F: Multimodal analgesia for postoperative pain control. J Clin Anesth 2001, 13:524-539.

7. Capdevila X, Barthelet Y, Biboulet P, Ryckwaert Y, Rubenovitch J, d'Athis F: Effects of perioperative analgesic technique on the surgical outcome and duration of rehabilitation after major knee surgery. Anesthesiology 1999, 91:8-15

8. Choi P, Bhandari M, Scott J, Douketis James D: Epidural analgesia for pain relief following hip or knee replacement. Cochrane Database Syst Rev 2003, 3, CD003071.

9. Liu SS, Richman JM, Thirlby RC, Wu CL: Efficacy of continuous wound catheters delivering local anesthetic for postoperative analgesia: a quantitative and qualitative systematic review of randomized controlled trials. J Am Coll Surg 2006, 203:914-932.

10. Bianconi M, Ferraro L, Traina GC, Zanoli G, Antonelli T, Guberti A, Ricci R, Massari L: Pharmacokinetics and efficacy of ropivacaine continuous wound instillation after joint replacement surgery. Br J Anaesth 2003, 91:830-835 
11. Wylde V, Gooberman-Hill R, Horwood J, Beswick A, Noble S, Brookes S, Smith AJ, Pyke M, Dieppe P, Blom AW: The effect of local anaesthetic wound infiltration on chronic pain after lower limb joint replacement: a protocol for a double-blind randomised controlled trial. BMC Musculoskelet Disord 2011, 12:53

12. Ventham NT, Hughes M, O'Neill S, Johns N, Brady RR, Wigmore SJ: Systematic review and meta-analysis of continuous local anaesthetic wound infiltration versus epidural analgesia for postoperative pain following abdominal surgery. Br J Surg 2013, 100:1280-1289.

13. Kjaergaard M, Moiniche S, Olsen KS: Wound infiltration with local anesthetics for post-operative pain relief in lumbar spine surgery: a systematic review. Acta Anaesthesiol Scand 2012, 56:282-290.

14. Higgins JPT, Green S: Cochrane Handbook for Systematic Reviews of Interventions, Version 5.0.2. The Cochrane Collaboration and John Wiley \& Sons: Chichester, West Sussex; 2008.

15. Moher D, Liberati A, Tetzlaff J, Altman DG: Preferred reporting items for systematic reviews and meta-analyses: the PRISMA statement. J Clin Epidemiol 2009, 62:1006-1012.

16. Andersen KV, Pfeiffer-Jensen M, Haraldsted V, Soballe K: Reduced hospital stay and narcotic consumption, and improved mobilization with local and intraarticular infiltration after hip arthroplasty: a randomized clinical trial of an intraarticular technique versus epidural infusion in 80 patients. Acta Orthop 2007, 78:180-186.

17. Kehlet H, Liu SS: Continuous local anesthetic wound infusion to improve postoperative outcome - Back to the periphery? Anesthesiology 2007 107:369-371.

18. Toftdahl K, Nikolajsen L, Haraldsted V, Madsen F, Tonnesen EK, Soballe K: Comparison of peri- and intraarticular analgesia with femoral nerve block after total knee arthroplasty: a randomized clinical trial. Acta Orthop 2007, 78:172-179.

19. Kerr DR, Kohan L: Local infiltration analgesia: a technique for the contro of acute postoperative pain following knee and hip surgery: a case study of 325 patients. Acta Orthop 2008, 79:174-183.

20. Sterne JA, Sutton AJ, loannidis JP, Terrin N, Jones DR, Lau J, Carpenter J, Rücker G, Harbord RM, Schmid CH, Tetzlaff J, Deeks JJ, Peters J, Macaskill P, Schwarzer G, Duval S, Altman DG, Moher D, Higgins JPT: Recommendations for examining and interpreting funnel plot asymmetry in meta-analyses of randomised controlled trials. BMJ 2011, 343:d4002.

21. Walter SD, Yao X: Effect sizes can be calculated for studies reporting ranges for outcome variables in systematic reviews. J Clin Epidemio/ 2007, 60:849-852.

22. Hedges LV, Olkin I: Statistical Methods for Meta-Analysis. San Diego: Academic Press; 1985.

23. Scholten RJPM, de Beurs E, Bouter LM: From effect size into number needed to treat. Lancet 1999, 354:598.

24. Higgins JP, White IR, Anzures-Cabrera J: Meta-analysis of skewed data: combining results reported on log-transformed or raw scales. Stat Med 2008, 27:6072-6092.

25. Bradburn MJ, Deeks JJ, Berlin JA, Russell Localio A: Much ado about nothing: a comparison of the performance of meta-analytical methods with rare events. Stat Med 2007, 26:53-77.

26. Higgins JP, Thompson SG: Quantifying heterogeneity in a meta-analysis. Stat Med 2002, 21:1539-1558.

27. Andersen $L$, Poulsen T, Krogh B, Nielsen T: Postoperative analgesia in total hip arthroplasty: a randomized double-blinded, placebo-controlled study on peroperative and postoperative ropivacaine, ketorolac, and adrenaline wound infiltration. Acta Orthop 2007, 78:187-192.

28. Parvataneni HK, Shah VP, Howard H, Cole N, Ranawat AS, Ranawat CS: Controlling pain after total hip and knee arthroplasty using a multimodal protocol with local periarticular injections. A prospective randomized study. J Arthroplasty 2007, 22:33-38.

29. Lee K-J, Min B-W, Bae K-C, Cho C-H, Kwon D-H: Efficacy of multimodal pain control protocol in the setting of total hip arthroplasty. Clin Orthop Surg 2009, 1:155-160.

30. Busch CA, Whitehouse MR, Shore BJ, MacDonald SJ, McCalden RW, Bourne $\mathrm{RB}$ : The efficacy of periarticular multimodal drug infiltration in total hip arthroplasty. Clin Orthop Relat Res 2010, 468:2152-2159.

31. Lu ZD, Li P: Analgesic effect of periarticular Ropivacaine infiltration and cyclooxygenase-2 inhibitor following total hip arthroplasty. J Clin Rehab Tissue Eng Res 2010, 14:7991-7994.
32. Liu W, Cong R, Li X, Wu Y, Wu H: Reduced opioid consumption and improved early rehabilitation with local and intraarticular cocktail analgesic injection in total hip arthroplasty: a randomized controlled clinical trial. Pain Med 2011, 12:387-393.

33. Lunn TH, Husted $H$, Solgaard S, Kristensen BB, Otte KS, Kjersgaard AG, Gaarn-Larsen L, Kehlet H: Intraoperative local infiltration analgesia for early analgesia after total hip arthroplasty: A randomized, double-blind, placebo-controlled trial. Reg Anesth Pain Med 2011, 36:424-429.

34. Aguirre J, Baulig B, Dora C, Ekatodramis G, Votta-Velis G, Ruland P, Borgeat A: Continuous epicapsular ropivacaine $0.3 \%$ infusion after minimally invasive hip arthroplasty: a prospective, randomized, double-blinded, placebo-controlled study comparing continuous wound infusion with morphine patient-controlled analgesia. Anesth Analg 2012, 114:456-461.

35. Dobie I, Bennett D, Spence DJ, Murray JM, Beverland DE: Periarticular local anesthesia does not improve pain or mobility after THA. Clin Orthop Relat Res 2012, 470:1958-1965.

36. Murphy TP, Byrne DP, Curtin P, Baker JF, Mulhall KJ: Can a periarticular levobupivacaine injection reduce postoperative opiate consumption during primary hip arthroplasty? Clin Orthop Relat Res 2012, 470:1151-1157.

37. Rikalainen-Salmi R, Forster JG, Makela K, Virolainen P, Leino KA, Pitkanen MT, Neuvonen PJ, Kuusniemi KS: Local infiltration analgesia with levobupivacaine compared with intrathecal morphine in total hip arthroplasty patients. Acta Anaesthesio/ Scand 2012, 56:695-705.

38. Busch CA, Shore BJ, Bhandari R, Ganapathy S, MacDonald SJ, Bourne RB, Rorabeck CH, McCalden RW: Efficacy of periarticular multimodal drug injection in total knee arthroplasty. A randomized trial. J Bone Joint Surg Am 2006, 88:959-963.

39. Vendittoli PA, Makinen P, Drolet P, Lavigne M, Fallaha M, Guertin MC, Varin F: A multimodal analgesia protocol for total knee arthroplasty. A randomized, controlled study. J Bone Joint Surg Am 2006, 88:282-289.

40. Han $C D$, Lee $D H$, Yang $H$ : Intra-synovial ropivacaine and morphine for pain relief after total knee arthroplasty: a prospective, randomized, double blind study. Yonsei Med J 2007, 48:295-300.

41. Zhang J, Jiang Y, Shao JJ, Shen H, Wang Q, Zhang XL: Effect of periarticular multimodal drug injection on pain after total knee arthroplasty. Chin J Clin Rehabil 2007, 11:8678-8682.

42. Fu P, Wu Y, Wu H, Li X, Qian Q, Zhu Y: Efficacy of intra-articular cocktail analgesic injection in total knee arthroplasty - A randomized controlled trial. Knee 2009, 16:280-284.

43. Krenzel BA, Cook C, Martin GN, Vail TP, Attarian DE, Bolognesi MP: Posterior capsular injections of ropivacaine during total knee arthroplasty: a randomized, double-blind, placebo-controlled study. J Arthroplasty 2009, 24:138-143.

44. Andersen KV, Bak M, Christensen BV, Harazuk J, Pedersen NA, Soballe K: A randomized, controlled trial comparing local infiltration analgesia with epidural infusion for total knee arthroplasty. Acta Orthop 2010, 81:606-610.

45. Carli F, Clemente A, Asenjo JF, Kim DJ, Mistraletti G, Gomarasca M, Morabito A, Tanzer M: Analgesia and functional outcome after total knee arthroplasty: periarticular infiltration vs continuous femoral nerve block. Br J Anaesth 2010, 105:185-195.

46. Essving P, Axelsson K, Kjellberg J, Wallgren O, Gupta A, Lundin A: Reduced morphine consumption and pain intensity with local infiltration analgesia (LIA) following total knee arthroplasty: a randomized double-blind study involving 48 patients. Acta Orthop 2010, 81:354-360.

47. Fu PL, Xiao J, Zhu YL, Wu HS, Li XH, Wu YL, Qian QR: Efficacy of a multimodal analgesia protocol in total knee arthroplasty: a randomized, controlled trial. J Int Med Res 2010, 38:1404-1412.

48. Spreng UJ, DahI V, Hjall A, Fagerland MW, Ræder J: High-volume local infiltration analgesia combined with intravenous or local ketorolac + morphine compared with epidural analgesia after total knee arthroplasty. Br J Anaesth 2010, 105:675-682.

49. Thorsell M, Holst $\mathrm{P}$, Hyldahl HC, Weidenhielm L: Pain control after total knee arthroplasty: a prospective study comparing local infiltration anesthesia and epidural anesthesia. Orthopedics 2010, 33:75-80.

50. Affas F, Nygårds E-B, Stiller C-O, Wretenberg P, Olofsson C: Pain control after total knee arthroplasty: a randomized trial comparing local infiltration anesthesia and continuous femoral block. Acta Orthop 2011, 82:441-447.

51. Essving P, Axelsson K, Aberg E, Spannar H, Gupta A, Lundin A: Local infiltration analgesia versus intrathecal morphine for postoperative pain 
management after total knee arthroplasty: a randomized controlled trial. Anesth Analg 2011, 113:926-933.

52. Chen Y, Zhang Y, Zhu YL, Fu P: Efficacy and safety of an intra-operative intra-articular magnesium/ropivacaine injection for pain control following total knee arthroplasty. J Int Med Res 2012, 40:2032-2040.

53. Koh IJ, Kang YG, Chang CB, Do SH, Seong SC, Kim TK: Does periarticular injection have additional pain relieving effects during contemporary multimodal pain control protocols for TKA?: a randomised, controlled study. Knee 2012, 19:253-259.

54. Mahadevan D, Walter RP, Minto G, Gale TC, McAllen CJ, Oldman M: Combined femoral and sciatic nerve block vs combined femoral and periarticular infiltration in total knee arthroplasty. A randomized controlled trial. J Arthroplasty 2012, 27:1806-1811.

55. Meftah M, Wong AC, Nawabi DH, Yun RJ, Ranawat AS, Ranawat CS: Pain management after total knee arthroplasty using a multimodal approach. Orthopedics 2012, 35:e660-e664.

56. Ng FY, Ng JKF, Chiu KY, Yan CH, Chan CW: Multimodal periarticular injection vs continuous femoral nerve block after total knee arthroplasty. A prospective, crossover, randomized clinical trial. J Arthroplasty 2012, 27:1234-1238.

57. Egger M, Smith GD, O'Rourke K: Introduction: rationale, potentials, and promise of systematic reviews. In Systematic Reviews in Health Care. London: BMJ Publishing Group; 2008:1-19.

58. Schmidt LM, Gotzsche PC: Of mites and men: reference bias in narrative review articles: a systematic review. J Fam Pract 2005, 54:334-338.

59. Sterne JA, Gavaghan D, Egger M: Publication and related bias in meta-analysis: power of statistical tests and prevalence in the literature. J Clin Epidemiol 2000, 53:1119-1129.

60. Wolfe F, Michaud K: Assessment of pain in rheumatoid arthritis: minimal clinically significant difference, predictors, and the effect of anti-tumor necrosis factor therapy. I Rheumatol 2007, 34:1674-1683.

61. Tashjian RZ, Deloach J, Porucznik CA, Powell AP: Minimal clinically important differences (MCID) and patient acceptable symptomatic state (PASS) for visual analog scales (VAS) measuring pain in patients treated for rotator cuff disease. J Shoulder Elbow Surg 2009, 18:927-932.

62. Hawker GA, Mian S, Kendzerska T, French M: Measures of adult pain: Visual Analog Scale for Pain (VAS Pain), Numeric Rating Scale for Pain (NRS Pain), McGill Pain Questionnaire (MPQ), Short-Form McGill Pain Questionnaire (SF-MPQ), Chronic Pain Grade Scale (CPGS), Short Form-36 Bodily Pain Scale (SF-36 BPS), and Measure of Intermittent and Constant Osteoarthritis Pain (ICOAP). Arthritis Care Res 2011, 63:S240-S252.

63. Tang R, Evans H, Chaput A, Kim C: Multimodal analgesia for hip arthroplasty. Orthop Clin North Am 2009, 40:377-387.

64. Auroy Y, Benhamou D, Bargues L, Ecoffey C, Falissard B, Mercier FJ, Bouaziz H, Samii K: Major complications of regional anesthesia in France: the SOS regional anesthesia hotline service. Anesthesiology 2002, 97:1274-1280.

65. Sharma S, Iorio R, Specht L, Davies-Lepie S, Healy W: Complications of femoral nerve block for total knee arthroplasty. Clin Orthop Relat Res 2010, 468:135-140.

66. Perkins FM, Kehlet $\mathrm{H}$ : Chronic pain as an outcome of surgery. A review of predictive factors. Anesthesiology 2000, 93:1 123-1133.

67. Macrae WA: Chronic post-surgical pain: 10 years on. Br J Anaesth 2008, 101:77-86.

68. Lau J, Antman EM, Jimenez-Silva J, Kupelnick B, Mosteller F, Chalmers TC Cumulative meta-analysis of therapeutic trials for myocardial infarction. New Engl J Med 1992, 327:248-254.

69. Kuchálik J, Granath B, Ljunggren A, Magnuson A, Lundin A, Gupta A: Postoperative pain relief after total hip arthroplasty: a randomized, double-blind comparison between intrathecal morphine and local infiltration analgesia. Br J Anaesth 2013, 111:793-799.

70. Nakamura T, Nakai T, Onishi A, Hashimoto K: A study of the usefulness of a periarticular multimodal drug cocktail injection for pain management after total hip arthroplasty. J Orthop 2013, 10:5-7.

71. Pandazi A, Kanellopoulos I, Kalimeris K, Batistaki C, Nikolakopoulos N, Matsota P, Babis GC, Kostopanagiotou G: Periarticular infiltration for pain relief after total hip arthroplasty: a comparison with epidural and PCA analgesia. Arch Orthop Trauma Surg 2013, 133:1607-1612.

72. Tammachote N, Kanitnate S, Manuwong S, Yakumpor T, Panichkul P: Is pain after TKA better with periarticular injection or intrathecal morphine? Clin Orthop Relat Res 2013, 471:1992-1999.
73. Andersen KV, Nikolajsen L, Haraldsted V, Odgaard A, Soballe K: Local infiltration analgesia for total knee arthroplasty: should ketorolac be added? Br J Anaesth 2013, 111:242-248.

74. Kelley TC, Adams MJ, Mulliken BD, Dalury DF: Efficacy of multimodal perioperative analgesia protocol with periarticular medication injection in total knee arthroplasty: a randomized, double-blinded study. J Arthroplasty 2013, 28:1274-1277.

75. Yue DB, Wang BL, Liu KP, Guo WS: Efficacy of multimodal cocktail periarticular injection with or without steroid in total knee arthroplasty. Chin Med J 2013, 126:3851-3855.

76. Chinachoti T, Lungnateetape A, Raksakietisak M: Periarticular infiltration of $0.25 \%$ bupivacaine on top of femoral nerve block and intrathecal morphine improves quality of pain control after total knee arthroplasty: a randomized double-blind placebo controlled clinical trial. J Med Assoc Thai 2012, 95:1536-1542.

77. Ashraf A, Raut W, Canty SJ, McLauchlan GJ: Pain control after primary total knee replacement. A prospective randomised controlled trial of local infiltration versus single shot femoral nerve block. Knee 2013, 20:324-327.

78. Chaumeron A, Audy D, Drolet P, Lavigne M, Vendittoli PA: Periarticular injection in knee arthroplasty improves quadriceps function. Clin Orthop Relat Res 2013, 471:2284-2295.

79. Moghtadaei M, Farahini H, Reza Faiz H, Mokarami F, Nabi R: Local infiltration analgesia; an effective method for pain relief and patient's satisfaction after total knee arthroplasty: a randomized clinical trial. Tehran Univ Med J 2013, 71:429-436.

80. YaDeau JT, Goytizolo EA, Padgett DE, Liu SS, Mayman DJ, Ranawat AS, Rade $M C$, Westrich GH: Analgesia after total knee replacement: local infiltration versus epidural combined with a femoral nerve blockade: a prospective, randomised pragmatic trial. Bone Joint J 2013, 95-B:629-635.

81. Gupta A: Wound infiltration with local anaesthetics in ambulatory surgery. Curr Opin Anaesthesiol 2010, 23:708-713.

doi:10.1186/1471-2474-15-220

Cite this article as: Marques et al: Local anaesthetic infiltration for peri-operative pain control in total hip and knee replacement: systematic review and meta-analyses of short- and long-term effectiveness. BMC Musculoskeletal Disorders 2014 15:220.

\section{Submit your next manuscript to BioMed Central and take full advantage of:}

- Convenient online submission

- Thorough peer review

- No space constraints or color figure charges

- Immediate publication on acceptance

- Inclusion in PubMed, CAS, Scopus and Google Scholar

- Research which is freely available for redistribution 